Proceedings of the Edinburgh Mathematical Society (2005) 48, 175-197 (C)

DOI:10.1017/S001309150300018X Printed in the United Kingdom

\title{
CLASSES OF OPERATOR-SMOOTH FUNCTIONS. III STABLE FUNCTIONS AND FUGLEDE IDEALS
}

\author{
EDWARD KISSIN ${ }^{1}$ AND VICTOR S. SHULMAN ${ }^{1,2}$ \\ ${ }^{1}$ Department of Computing, Communications Technology and Mathematics, \\ London Metropolitan University, 166-220 Holloway Road, \\ London N7 8DB, UK (e.kissin@londonmet.ac.uk) \\ ${ }^{2}$ Department of Mathematics, Vologda State Technical University, \\ Vologda, Russia (shulman_v@yahoo.com)
}

(Received 8 March 2003)

\begin{abstract}
This paper continue to study the interrelation and hierarchy of the spaces of operatorLipschitz functions and the spaces of functions closed to them: commutator bounded and operator stable. It also examines various properties of symmetrically normed ideals, introduces new classes of ideals: regular and Fuglede, and investigates them.
\end{abstract}

Keywords: operator; Lipschitz; functions; symmetrically normed ideals

2000 Mathematics subject classification: Primary 47A56; 47L20

\section{Introduction}

This paper is a sequel to the article [17] on operator-Lipschitz functions. It continues to study operator-Lipschitz functions and functions close to them: commutator bounded and operator stable functions. It also examines various properties of symmetrically normed ideals. New classes of ideals (regular and Fuglede) are introduced and investigated in the paper. A summary of the main results for the most important class of Schatten ideals is given at the end of $\S 5$. We refer the reader to [17] for a review of the history of the subject, the impetus behind our study, general notation and various technical results.

Recall that we denote by $B(H)$ the algebra of all bounded operators on a Hilbert space $H$, by $C(H)$ the ideal of all compact operators, and by $\mathcal{F}$ the ideal of all finiterank operators in $B(H)$. A two-sided ideal $J$ of $B(H)$ is symmetrically normed (see [12]) if it is a Banach space with respect to a norm $\|\cdot\|_{J}$ and

$$
\|A X B\|_{J} \leqslant\|A\|\|X\|_{J}\|B\| \quad \text { for } A, B \in B(H) \text { and } X \in J .
$$

It is a $*$-ideal and, by the Calkin theorem, $\mathcal{F} \subset J \subseteq C(H)$. We denote by $\alpha$ compact subsets of $\mathbb{C}$ and set

$$
J_{\text {nor }}(\alpha)=\{A \in J: A \text { is normal and } \operatorname{Sp}(A) \subseteq \alpha\} .
$$


Any continuous function $g$ on $\alpha$ defines a map $T \rightarrow g(T)$ from $J_{\text {nor }}(\alpha)$ to $B(H)$. Various smoothness conditions when imposed on this map characterize important classes of operator-smooth functions. The condition that it is Lipschitzian, for example, defines the class of $J$-Lipschitz functions. Thus a function $g$ is called $J$-Lipschitzian on $\alpha$ if there is $D>0$ such that, for $A, B \in J_{\text {nor }}(\alpha)$,

$$
g(A)-g(B) \in J \quad \text { and } \quad\|g(A)-g(B)\|_{J} \leqslant D\|A-B\|_{J} .
$$

We denote by $J$ - $\operatorname{Lip}(\alpha)$ the space of all $J$-Lipschitz functions on $\alpha$.

A function $g$ is called commutator $J$-bounded on $\alpha$ if there is $D>0$ such that, for $A \in J_{\text {nor }}(\alpha)$ and $X \in B(H)$,

$$
g(A) X-X g(A) \in J \quad \text { and } \quad\|g(A) X-X g(A)\|_{J} \leqslant D\|A X-X A\|_{J} .
$$

We denote by $J-\operatorname{CB}(\alpha)$ the space of all commutator $J$-bounded functions on $\alpha$.

In this paper we continue the study of intrinsic properties, interrelation and hierarchy of the spaces $J$ - $\operatorname{Lip}(\alpha)$ and $J-\operatorname{CB}(\alpha)$, and consider also another class of functions close to them: operator $J$-stable functions.

It was shown in $[\mathbf{1 7}]$ that the space $J$ - $\operatorname{Lip}(\alpha)$ always contains $J-\operatorname{CB}(\alpha)$ and that these spaces coincide if and only if $\alpha$ is a $J$-Fuglede set, that is, there is $C>0$ such that

$$
\left\|A^{*} X-X A^{*}\right\|_{J} \leqslant C\|A X-X A\|_{J}, \quad \text { for } A \in J_{\text {nor }}(\alpha) \text { and } X \in B(H) .
$$

For example, all compact sets in $\mathbb{R}$ are $J$-Fuglede for all s.n. ideals $J$ and for $J=B(H)$. The possibility to reduce the study of $J$-Lipschitz functions to the study of commutator $J$-bounded functions is very important, since it enables us to use the powerful techniques of the interpolation theory to compare the spaces $J$ - $\operatorname{Lip}(\alpha)$ for different ideals. The conditions under which a compact subset of $\mathbb{C}$ is a $J$-Fuglede set were studied in $[\mathbf{1 7}]$ and will be further investigated here.

If all compact subsets of $\mathbb{C}$ are $J$-Fuglede, or, equivalently, if the unit disc is $J$-Fuglede, then $J$ is called a Fuglede ideal. So Fuglede ideals are the ideals $J$ for which $J$ - $\operatorname{Lip}(\alpha)=$ $J-\mathrm{CB}(\alpha)$ for all compacts $\alpha$ in $\mathbb{C}$.

In $\S 3$ we obtain some sufficient condition for an ideal $J$ to be Fuglede. We show that if the Boyd indices (see $[\mathbf{2}]$ ) of $J$ lie in $(1, \infty)$, then $J$ is Fuglede. This extends the results of Weiss [25], Abdessemed and Davies [1] and Shulman [24] that all $\mathfrak{S}^{p}, p \in(1, \infty)$, are Fuglede ideals. It also shows that Lorentz ideals $\mathfrak{S}^{r, p}$ are Fuglede if $1<p$. We establish that $\mathfrak{S}^{1}$ and $\mathfrak{S}^{\infty}$ are not Fuglede ideals (the fact that $\mathfrak{S}^{b}$ is not Fuglede follows from Corollary 3.3 of $[\mathbf{1 5}])$.

We also use Hadamard multipliers and the interpolation theory to compare the spaces $J-\mathrm{CB}(\alpha)$ for various ideals $J$. We prove that if the Boyd indices of $J$ lie between $p$ and $p /(p-1)$, then $\mathfrak{S}^{p}-\mathrm{CB}(\alpha) \subseteq J$ - $\mathrm{CB}(\alpha)$. This implies, in particular, that $\mathfrak{S}^{p}-\mathrm{CB}(\alpha) \subseteq \mathfrak{S}^{q}-\mathrm{CB}(\alpha)$ when

$$
\min \left(p, \frac{p}{p-1}\right) \leqslant q \leqslant \max \left(p, \frac{p}{p-1}\right) .
$$


The normal operators $A, B$ in the definitions of $J$-Lipschitz and of commutator $J$ bounded functions in (1.2) and (1.3) belong to $J$. The question arises as to whether these inequalities hold for all normal $A, B \in B(H)$ with spectra in $\alpha$. Using the theory of complex interpolation and the results of Bercovici and Voiculescu in [3] on quasidiagonalization of operators modulo ideals, we obtain in $\S 4$ that 'extended' inequalities (1.2) and (1.3) hold for all separable ideals and their duals. This generalizes the result of Kittaneh [18], who considered the case $J=\mathfrak{S}^{2}$. We use these results in $\S 5$ to study $J$-stable functions.

A function $g$ on $\alpha$ is called $J$-stable if the condition $A-B \in J$ implies $g(A)-g(B) \in J$ for all $A, B \in J_{\text {nor }}(\alpha)$. The property of $J$-stability of functions is important for various applications in mathematical physics. It was studied by Daletskii and Krein [8], Birman and Solomyak [6], Farforovskaya [10], Peller [22] and others. For separable ideals and their duals (see Corollary 4.6) this property of functions is, generally speaking, weaker than the property to be $J$-Lipschitzian. In $\S 5$ we show that in many important cases ( $J=\mathfrak{S}^{p}, 1<p<\infty$, for example) they are equivalent.

Johnson and Williams [15] constructed a normal operator $A$ and a bounded $X$ such that $[A, X] \in \mathfrak{S}^{1}$ and $\left[A^{*}, X\right] \notin \mathfrak{S}^{1}$. Weiss $[\mathbf{2 5}]$ asked whether for compact $X$, the condition $[A, X] \in \mathfrak{S}^{1}$ always implies $\left[A^{*}, X\right] \in \mathfrak{S}^{1}$. A negative answer to this question was given in [23]. It was shown in [16] that, for any $p>1$, one can find $X \in \mathfrak{S}^{p}$ such that $[A, X] \in \mathfrak{S}^{1}$ and $\left[A^{*}, X\right] \notin \mathfrak{S}^{1}$. In $\S 5$ we construct a normal compact operator $A$ and a compact operator $X$ such that $[A, X] \in \mathfrak{S}^{1}$, while $\left[A^{*}, X\right] \notin \mathfrak{S}^{1}$.

\section{Preliminaries}

Let $c_{0}$ be the space of all sequences of real numbers converging to 0 , let $\hat{c}$ be the subspace of $c_{0}$ of sequences with a finite number of non-zero elements, and let $\Phi$ be the set of all symmetric norming (s.n.) functions on $\hat{c}$ (see [12, $\S$ III.3]). For $\xi=\left\{\xi_{i}\right\} \in c_{0}$, set $\xi^{(n)}=\left\{\xi_{1}, \ldots, \xi_{n}, 0, \ldots\right\}$. Then $\xi^{(n)} \in \hat{c}$. For $\phi \in \Phi$, the sequence $\phi\left(\xi^{(n)}\right)$ does not decrease. Set $\phi(\xi)=\lim \phi\left(\xi^{(n)}\right)$ and $c^{\phi}=\left\{\xi \in c_{0}: \phi(\xi)<\infty\right\}$.

For $A \in C(H)$, let $s(A)=\left\{s_{i}(A)\right\}$ be the non-increasing sequence of all eigenvalues of $\left(A^{*} A\right)^{1 / 2}$ repeated according to multiplicity. For $\phi \in \Phi$, the set $J^{\phi}=\{A \in C(H)$ : $\left.s(A) \in c^{\phi}\right\}$ with norm $\|A\|_{J^{\phi}}=\phi(s(A))$ is an s.n. ideal. The closure $J_{0}^{\phi}$ of $\mathcal{F}$ in $\|\cdot\|_{J^{\phi}}$ is a separable ideal and $J_{0}^{\phi} \subseteq J^{\phi}$. An s.n. ideal is separable if and only if it coincides with some $J_{0}^{\phi}$ (see [12]). For some $\phi, J^{\phi}=J_{0}^{\phi}$. An important class of such functions consists of

$$
\phi_{p}(\xi)=\left(\sum_{i=1}^{\infty}\left|\xi_{i}\right|^{p}\right)^{1 / p} \quad \text { for } 1 \leqslant p<\infty, \quad \text { and } \quad \phi_{\infty}(\xi)=\sup \left\|\xi_{i}\right\| .
$$

The corresponding ideals $\mathfrak{S}^{p}$ are called Schatten ideals. We denote $C(H)$ by $\mathfrak{S}^{\infty}$ and $B(H)$ by $\mathfrak{S}^{b}$.

For $\phi \in \Phi$, there is the adjoint function $\phi^{*}$ such that the ideal $J^{\phi^{*}}$ is isomorphic to the dual space of $J_{0}^{\phi}$ : any bounded functional on $J_{0}^{\phi}$ has the form

$$
F(X)=\operatorname{Tr}(X T)=\operatorname{Tr}(T X), \quad \text { where } T \in J^{\phi^{*}} \text { and }\|F\|=\|T\|_{J^{\phi^{*}}} .
$$


Let

$$
p^{\prime}= \begin{cases}\frac{p}{p-1}, & \text { for } 1<p<\infty, \\ 1, & \text { for } p=\infty, \\ b, & \text { for } p=1 .\end{cases}
$$

Then $\phi_{p^{\prime}}=\left(\phi_{p}\right)^{*}$, so $\mathfrak{S}^{p^{\prime}}$ is isometrically isomorphic to the dual space of $\mathfrak{S}^{p}$.

The results below are known. We include their proof for the reader's convenience.

Proposition 2.1. Let $J$ and $I$ be s.n. ideals.

(i) If $J \subseteq I$, then there exists $c>0$ such that $\|X\|_{I} \leqslant c\|X\|_{J}$ for $X \in J$.

(ii) There is a unique, up to equivalence (see [12, III.3.4]), function $\phi \in \Phi$ such that $J_{0}^{\phi} \subseteq J \subseteq J^{\phi}$. The norms $\|\cdot\|_{J^{\phi}},\|\cdot\|_{J}$ coincide on $J_{0}^{\phi}$ and $\|T\|_{J^{\phi}} \leqslant\|T\|_{J}$ for $T \in J$.

(iii) If $J$ is reflexive, then there is $\phi \in \Phi$ such that $J_{0}^{\phi}=J=J^{\phi}$.

Proof. Consider the norm $\|X\|^{\sim}=\max \left(\|X\|_{J},\|X\|_{I}\right)$ on $J$. Since $\|X\| \leqslant\|X\|_{J}$ and $\|X\| \leqslant\|X\|_{I}$, for $X \in J$, one can easily check that $\left(J,\|\cdot\|^{\sim}\right)$ is a Banach space. The identity operator from $\left(J,\|\cdot\|^{\sim}\right)$ to $\left(J,\|\cdot\|_{J}\right)$ is bounded. By Banach's theorem, the inverse is also bounded, so there is $c>0$ such that $\|X\|_{I} \leqslant c\|X\|_{J}$. Part (i) is proved.

Consider $\|\cdot\|_{J}$ on $\mathcal{F}$. It follows from III.3.1 of [12] that there is $\phi \in \Phi$ such that $\|X\|_{J}=\|X\|_{J^{\phi}}$, for $X \in \mathcal{F}$. Hence $J_{0}^{\phi} \subseteq J$ and the norms $\|\cdot\|_{J},\|\cdot\|_{J^{\phi}}$ coincide on $J_{0}^{\phi}$. Let finite-dimensional projections $P_{n}$ strongly converge to 1 . For $T \in J$, the operators $P_{n} T$ belong to $\mathcal{F}$, strongly converge to $T$ and

$$
\left\|P_{n} T\right\|_{J^{\phi}}=\left\|P_{n} T\right\|_{J} \leqslant\left\|P_{n}\right\|\|T\|_{J}=\|T\|_{J} .
$$

By Theorem III.5.1 of [12], $T \in J^{\phi}$ and $\|T\|_{J^{\phi}} \leqslant\|T\|_{J}$. Thus $J_{0}^{\phi} \subseteq J \subseteq J^{\phi}$.

Suppose that also $J_{0}^{\psi} \subseteq J \subseteq J^{\psi}$. By (i), there are $c_{1}, c_{2}>0$ such that $\|X\|_{J^{\psi}} \leqslant$ $c_{1}\|X\|_{J}$, for $X \in J$, and $\|X\|_{J} \leqslant c_{2}\|X\|_{J^{\psi}}$ for $X \in J_{0}^{\psi}$. Hence the norms $\|\cdot\|_{J}$ and $\|\cdot\|_{J^{\psi}}$ are equivalent on $\mathcal{F}$, so the norms $\|\cdot\|_{J^{\phi}}$ and $\|\cdot\|_{J^{\psi}}$ are equivalent on $\mathcal{F}$. Thus (see [12, III.4.2]) $\phi, \psi$ are equivalent and $J^{\phi}=J^{\psi}$. Part (ii) is proved.

Let $J$ be reflexive. By (ii), $J_{0}^{\phi} \subseteq J \subseteq J^{\phi}$. Since $J_{0}^{\phi}$ is a closed subspace of $J, J_{0}^{\phi}$ is also reflexive (see [13, Proposition 67$]$ ). Since $J^{\phi^{*}}$ is the dual of $J_{0}^{\phi}$, it is reflexive. Hence its closed subspace $J_{0}^{\phi^{*}}$ is also reflexive. Since $J^{\phi}$ is the dual of $J_{0}^{\phi^{*}}$, the ideal $J_{0}^{\phi^{*}}$ is the dual of $J^{\phi}$. If the dual of a Banach space is separable, the space itself is separable. Thus, since $J_{0}^{\phi^{*}}$ is separable, $J^{\phi}$ is separable, so $J_{0}^{\phi}=J=J^{\phi}$.

\section{Boyd indices and Fuglede ideals}

Let $J \subset I$ be s.n. ideals. A linear operator $T$ on $I$ is called bounded on $(J, I)$ if it is bounded on $I$, preserves $J$ and its restriction $\left.T\right|_{J}$ to $J$ is bounded in $\|\cdot\|_{J}$. We will denote by $\|T\|_{I}$ and $\|T\|_{J}$ the norms of the operator $T$ on $I$ and $J$. The set $L(J, I)$ of all bounded operators on $(J, I)$ is a Banach space with norm $\max \left(\|T\|_{I},\|T\|_{J}\right)$. We consider (in a simple form needed for our purposes) the notion of an interpolation space (see [19]). 
Definition 3.1. Let $J \subset I$ be s.n. ideals. A Banach space $\left(K,\|\cdot\|_{K}\right), J \subset K \subset I$, is called an interpolation space for the pair $(J, I)$ if any bounded operator $T$ on $(J, I)$ preserves $K$ and the restriction $\left.T\right|_{K}$ is a bounded operator on $K$.

Lemma 3.2. Let $\left(K,\|\cdot\|_{K}\right)$ be an interpolation space for a pair $(J, I)$ of s.n. ideals.

(i) There is a norm $\|\cdot\|_{K}^{\prime}$ on $K$ equivalent to $\|\cdot\|_{K}$ such that $\left(K,\|\cdot\|_{K}^{\prime}\right)$ is an s.n. ideal.

(ii) If for each $T \in L(J, I),\|T\|_{K} \leqslant\|T\|_{I}^{t}\|T\|_{J}^{1-t}$ for some $t \in[0,1]$, then $\left(K,\|\cdot\|_{K}\right)$ is an s.n. ideal.

Proof. The map $\left.T \rightarrow T\right|_{K}$ from $L(J, I)$ into $B(K)$ is closed. By the closed graph theorem, there is $C>0$ such that

$$
\|T\|_{K} \leqslant C \max \left(\|T\|_{I},\|T\|_{J}\right) \text { for all } T \in L(J, I) .
$$

For $A \in B(H)$, the left and right multiplication operators $L_{A}, R_{A}$ preserve $I$ and $J$ and $\|A\|=\left\|L_{A}\right\|_{I}=\left\|L_{A}\right\|_{J}=\left\|R_{A}\right\|_{I}=\left\|R_{A}\right\|_{J}$. Therefore, $L_{A}$ and $R_{A}$ are bounded on $(J, I)$. Hence they preserve $K$, so that $K$ is an ideal.

For all $A, B \in B(H)$ and $X \in K$, it follows from (3.1) that

$$
\|A X B\|_{K}=\left\|L_{A} R_{B} X\right\|_{K} \leqslant C \max \left(\left\|L_{A} R_{B}\right\|_{I},\left\|L_{A} R_{B}\right\|_{J}\right)\|X\|_{K} \leqslant C\|A\|\|B\|\|X\|_{K} .
$$

Define a new norm on $K$ by the formula

$$
\|X\|_{K}^{\prime}=\sup \left\{\|A X B\|_{K}: A, B \in B(H),\|A\| \leqslant 1,\|B\| \leqslant 1\right\} .
$$

It is equivalent to $\|\cdot\|_{K}$, since $\|X\|_{K}=\|\mathbf{1} X \mathbf{1}\|_{K} \leqslant\|X\|_{K}^{\prime} \leqslant C\|X\|_{K}$. For $F, G \in B(H)$,

$$
\|F X G\|_{K}^{\prime}=\|F\|\|G\|\left\|\frac{F}{\|F\|} X \frac{G}{\|G\|}\right\|_{K}^{\prime} \leqslant\|F\|\|G\|\|X\|_{K}^{\prime} .
$$

Thus $\left(K,\|\cdot\|_{K}^{\prime}\right)$ is an s.n. ideal. Part (i) is proved.

For $A, B \in B(H)$ and $X \in K$, we have

$$
\begin{aligned}
\|A X B\|_{K} & =\left\|L_{A} R_{B} X\right\|_{K} \leqslant\left\|L_{A} R_{B}\right\|_{K}\|X\|_{K} \\
& \leqslant\left\|L_{A} R_{B}\right\|_{I}^{t}\left\|L_{A} R_{B}\right\|_{J}^{1-t}\|X\|_{K} \leqslant\left\|L_{A}\right\|_{I}^{t}\left\|R_{B}\right\|_{I}^{t}\left\|L_{A}\right\|_{J}^{1-t}\left\|R_{B}\right\|_{J}^{1-t}\|X\|_{K} \\
& =\|A\|^{t}\|B\|^{t}\|A\|^{1-t}\|B\|^{1-t}\|X\|_{K}=\|A\|\|B\|\|X\|_{K} .
\end{aligned}
$$

Thus $\left(K,\|\cdot\|_{K}\right)$ is an s.n. ideal.

Let $A=\operatorname{diag}\left(\lambda_{1}, \ldots, \lambda_{n}, \ldots\right)$ be a diagonal operator with respect to a basis $\left\{e_{n}\right\}$ and let a compact $\alpha$ in $\mathbb{C}$ contain all $\lambda_{n}$. For a continuous function $g$ on $\alpha$, set

$$
m_{i j}= \begin{cases}\frac{g\left(\lambda_{i}\right)-g\left(\lambda_{j}\right)}{\lambda_{i}-\lambda_{j}}, & \text { if } \lambda_{i} \neq \lambda_{j}, \\ 0, & \text { if } \lambda_{i}=\lambda_{j},\end{cases}
$$

and consider the matrix $M(A, g)=\left(m_{i j}\right)$. With each $X \in B(H)$ we associate the matrix $\left(x_{i j}\right)$, with $x_{i j}=\left(X e_{j}, e_{i}\right)$, and set $M(A, g) \circ X=\left(m_{i j} x_{i j}\right)$. The matrix $M(A, g)$ is called a Hadamard $J$-multiplier (see [19]), if $M(A, g) \circ X$ belongs to $J$ for each $X \in J$. 
Theorem 3.3. Let $\phi$ be an s.n. function, let $\phi^{*}$ be its adjoint, and suppose that $J_{0}^{\phi} \subset J^{\phi^{*}}$. Let $g$ be a commutator $J^{\phi}$-bounded function on $\alpha$. If an s.n. ideal $K$ is an interpolation space for the pair $\left(J_{0}^{\phi}, J^{\phi^{*}}\right)$, then there is $D>0$ such that

$$
\|[g(A), X]\|_{K} \leqslant D\|[A, X]\|_{K} \quad \text { for } A \in K_{\text {nor }}(\alpha) \text { and } X \in C(H) .
$$

If $K$ is $J^{\psi}$ or $J_{0}^{\psi}$, for some s.n. function $\psi$, then $g$ is commutator $K$-bounded.

Proof. By Corollary 5.4 of $[\mathbf{1 7}], g$ is commutator $J^{\phi^{*}}$-bounded on $\alpha$. Since $K \subset J^{\phi^{*}}$, it follows from Corollary 5.3 of $[\mathbf{1 7}]$ that, for all $A \in K_{\text {nor }}(\alpha)$, the matrices $M(A, g)$ are Hadamard $J^{\phi^{*}}$-multipliers and there is $\mathcal{D}>0$ such that $\|M(A, g)\|_{J^{\phi^{*}}} \leqslant \mathcal{D}$. By Lemma 5.1 of $[\mathbf{1 7}], M(A, g)$ are also Hadamard $J_{0}^{\phi}$-multipliers and $\|M(A, g)\|_{J_{0}^{\phi}} \leqslant \mathcal{D}$. Hence all $M(A, g)$ are bounded on the pair $\left(J_{0}^{\phi}, J^{\phi^{*}}\right)$. Since $K$ is an interpolation space for this pair, $M(A, g)$ are Hadamard $K$-multipliers. By $(3.1),\|M(A, g)\|_{K} \leqslant C \mathcal{D}$. Hence, by Proposition 5.2 of $[\mathbf{1 7}],\|[g(A), X]\|_{K} \leqslant D\|[A, X]\|_{K}$ for $A \in K_{\text {nor }}(\alpha), X \in K$ and $D=C \mathcal{D}$.

Let $X \in C(H)$. Choose $X_{n}$ in $K$ such that $\left\|X-X_{n}\right\| \rightarrow 0$. Since $g$ is commutator $J^{\phi^{*}}$-bounded on $\alpha$, it is $J^{\phi^{*}}$-Lipschitzian. Hence it is Lipschitzian on $\alpha$ and it follows from Lemma 3.1 of $[\mathbf{1 7}]$ that $g(A)-g(0) 1 \in K$. Hence

$$
\begin{aligned}
\|[g(A), X]\|_{K} & \leqslant\left\|\left[g(A), X_{n}\right]\right\|_{K}+\left\|\left[g(A)-g(0) \mathbf{1}, X-X_{n}\right]\right\|_{K} \\
& \leqslant D\left\|\left[A, X_{n}\right]\right\|_{K}+2\|g(A)-g(0) \mathbf{1}\|_{K}\left\|X-X_{n}\right\| .
\end{aligned}
$$

Since $\left\|\left[A, X_{n}\right]-[A, X]\right\|_{K} \leqslant 2\left\|X-X_{n}\right\|\|A\|_{K}$, we have $\left\|\left[A, X_{n}\right]\right\|_{K} \rightarrow\|[A, X]\|_{K}$. Therefore, $\|[g(A), X]\|_{K} \leqslant D\|[A, X]\|_{K}$.

If $I$ is $J^{\psi}$ or $J_{0}^{\psi}$, then, by Proposition 3.4 of [17], $g$ is commutator $K$-bounded on $\alpha$.

Corollary 3.4. Let $p \in\{1, \infty, b\}$. Every commutator $\mathfrak{S}^{p}$-bounded function $g$ on $\alpha$ is commutator $J^{\phi}$-bounded on $\alpha$ for each norming function $\phi$.

Proof. By Corollary 5.4 of $[\mathbf{1 7}], g$ is commutator $\mathfrak{S}^{1}$ - and $\mathfrak{S}^{\infty}$-bounded. Mityagin [21] obtained (see also Theorem 3.B in [5]) that any ideal $J^{\phi}$ is an interpolation space for the pair $\left(\mathfrak{S}^{1}, \mathfrak{S}^{\infty}\right)$. Applying Theorem 3.3, we complete the proof.

For further applications of Theorem 3.3 we consider the Boyd indices (see $[\mathbf{2}, \mathbf{2 0}]$ ) of s.n. ideals $J$. Set

$$
\beta_{J}^{*}(n)=\inf _{X \in J} \frac{\|\overbrace{X \oplus \cdots \oplus X}^{n}\|_{J}}{\|X\|_{J}} \text { and } \gamma_{J}^{*}(n)=\sup _{X \in J} \frac{\|\overbrace{X \oplus \cdots \oplus X}^{n}\|_{J}}{\|X\|_{J}}
$$

(see Remark 2.1 in $[\mathbf{1 7}]$ ). The Boyd indices of $J$ are defined by the formulae

$$
p_{J}=\sup _{n}\left(\frac{\ln (n)}{\ln \left(\gamma_{J}^{*}(n)\right)}\right) \quad \text { and } \quad q_{J}=\inf _{n}\left(\frac{\ln (n)}{\ln \left(\beta_{J}^{*}(n)\right)}\right) .
$$


For an s.n. function $\phi$, the symmetric sequence space $c_{0}^{\phi}$ is the closure of the space $\hat{c}$ with respect to the norm $\|\xi\|=\phi(\xi)$, for $\xi \in \hat{c}$. Clearly, $c_{0}^{\phi} \subseteq c^{\phi}$ (see $\S 2$ ). Consider the dilation maps $D_{n}$ on $c_{0}^{\phi}$ defined by the formula

$$
D_{n}(\xi)=(\overbrace{\xi_{1}, \ldots, \xi_{1}}^{n}, \overbrace{\xi_{2}, \ldots, \xi_{2}}^{n}, \ldots, \overbrace{\xi_{k}, \ldots, \xi_{k}}^{n}, \ldots)
$$

and their left inverses

$$
D_{1 / n}(\xi)=\frac{1}{n}\left(\sum_{i=1}^{n} \xi_{i}, \sum_{i=n+1}^{2 n} \xi_{i}, \ldots, \sum_{i=(k-1) n+1}^{k n} \xi_{i}, \ldots\right),
$$

where $\xi=\left(\xi_{1}, \xi_{2}, \ldots, \xi_{k}, \ldots\right)$. The Boyd indices of $c_{0}^{\phi}$ are defined by the formulae

$$
\begin{aligned}
& p_{\phi}=\sup _{n}\left(\frac{\ln (n)}{\ln \left\|D_{n}\right\|}\right)=\lim _{n \rightarrow \infty}\left(\frac{\ln (n)}{\ln \left\|D_{n}\right\|}\right), \\
& q_{\phi}=\inf _{n}\left(\frac{\ln (1 / n)}{\ln \left\|D_{1 / n}\right\|}\right)=\lim _{n \rightarrow \infty}\left(\frac{\ln (1 / n)}{\ln \left\|D_{1 / n}\right\|}\right) .
\end{aligned}
$$

The norm $\left\|D_{1 / n}\right\|$ can be expressed in terms of the operator $D_{n}$ :

$$
\left\|D_{1 / n}\right\|^{-1}=\inf _{\xi \in c_{0}^{\phi}} \frac{\left\|D_{n}(\xi)\right\|}{\|\xi\|} .
$$

Indeed, let $\pi$ be the cyclic permutation modulo $n$ of the set of positive integers: $\pi(k)=$ $k+1$, if $k$ is not divisible by $n$, and $\pi(k)=k-n+1$, if $k$ is divisible by $n$. For $\xi=\left(\xi_{1}, \ldots, \xi_{k}, \ldots\right)$, set $\Pi(\xi)=\left(\xi_{\pi(1)}, \ldots, \xi_{\pi(k)}, \ldots\right)$. Then

$$
D_{1 / n} D_{n}(\xi)=\xi \quad \text { and } \quad D_{n} D_{1 / n}(\xi)=\frac{1}{n}\left(\xi+\Pi(\xi)+\Pi^{2}(\xi)+\cdots+\Pi^{n-1}(\xi)\right) .
$$

Since $\phi(\xi)=\|\xi\|=\left\|\Pi^{i}(\xi)\right\|$, for all $i$, we have $\left\|D_{n} D_{1 / n}(\xi)\right\| \leqslant\|\xi\|$. Denote by $\lambda$ the right-hand side expression in (3.4). Then

$$
\lambda \leqslant \inf _{\eta \in c_{0}^{\phi}} \frac{\left\|D_{n} D_{1 / n}(\eta)\right\|}{\left\|D_{1 / n}(\eta)\right\|} \leqslant \inf _{\eta \in c_{0}^{\phi}} \frac{\|\eta\|}{\left\|D_{1 / n}(\eta)\right\|}=\left(\sup _{\eta \in c_{0}^{\phi}} \frac{\left\|D_{1 / n}(\eta)\right\|}{\|\eta\|}\right)^{-1}=\left\|D_{1 / n}\right\|^{-1} .
$$

In order to finish the proof of (3.4), observe that, on the other hand,

$$
\begin{aligned}
\lambda & =\inf _{\xi \in c_{0}^{\phi}}\left(\frac{\left\|D_{1 / n} D_{n}(\xi)\right\|}{\left\|D_{n}(\xi)\right\|}\right)^{-1}=\left(\sup _{\xi \in c_{0}^{\phi}} \frac{\left\|D_{1 / n} D_{n}(\xi)\right\|}{\left\|D_{n}(\xi)\right\|}\right)^{-1} \\
& \geqslant\left(\sup _{\eta \in c_{0}^{\phi}} \frac{\left\|D_{1 / n}(\eta)\right\|}{\|\eta\|}\right)^{-1}=\left\|D_{1 / n}\right\|^{-1} .
\end{aligned}
$$

Let $J=J_{0}^{\phi}$ be separable. For $X \in J$, let $s(X)$ be the non-increasing sequence of the eigenvalues of the operator $\left(X^{*} X\right)^{1 / 2}$. Then $s: X \rightarrow s(X)$ maps $J$ onto $c_{0}^{\phi},\|X\|_{J}=$ $\|s(X)\|$ and

$$
s(\overbrace{X \oplus \cdots \oplus X}^{n})=D_{n}(s(X))
$$


Using this and (3.4) we obtain

$$
\left\|D_{1 / n}\right\|^{-1}=\inf _{X \in J} \frac{\left\|D_{n}(s(X))\right\|}{\|s(X)\|}=\beta_{J}^{*}(n) \quad \text { and } \quad\left\|D_{n}\right\|=\sup _{X \in J} \frac{\left\|D_{n}(s(X))\right\|}{\|s(X)\|}=\gamma_{J}^{*}(n) .
$$

Therefore, $p_{J_{0}^{\phi}}=p_{\phi}$ and $q_{J_{0}^{\phi}}=q_{\phi}$. We also have

$$
1 \leqslant\left\|D_{1 / n}\right\|^{-1} \leqslant\left\|D_{n}\right\| \leqslant n \quad \text { and } \quad\left\|D_{n+1}\right\| \leqslant\left\|D_{n}\right\|+1, \quad \text { so } p_{\phi} \leqslant q_{\phi} .
$$

If $J \neq \mathfrak{S}^{\infty}$, then $\left\|D_{n}\right\| \rightarrow \infty$, as $n \rightarrow \infty($ see $[\mathbf{1 2}$, III. $\S 3])$.

For $\mathfrak{S}^{r}$, with $r \in[1, \infty)$, we have $\left\|D_{1 / n}\right\|=\left\|D_{n}\right\|=n^{1 / r}$, so $p_{\mathfrak{S}^{r}}=q_{\mathfrak{S}^{r}}=r$.

The Lorentz space $l_{r, q}$, for $1 \leqslant r, q<\infty$, consists of all sequences $\xi=\left(\xi_{1}, \xi_{2}, \ldots\right) \in c_{0}$ such that

$$
\|\xi\|_{r, q}=\phi(\xi)=\phi\left(\xi^{*}\right)=\left(\sum_{k=1}^{\infty} k^{(r / q)-1}\left(\xi_{k}^{*}\right)^{r}\right)^{1 / r}<\infty
$$

where $\xi^{*}$ is the non-increasing rearrangement of $\left(\left|\xi_{1}\right|,\left|\xi_{2}\right|, \ldots\right)$. The Lorentz s.n. ideal $\mathfrak{S}^{r, q}$ (see [4]) consists of all compact operators $X$ such that $\|X\|_{r, q}=\phi(s(X))<\infty$.

Proposition 3.5. For $J=\mathfrak{S}^{r, q}, p_{J}=q_{J}=r$.

Proof. Set $\beta=(r / q)-1$ and $\lambda_{k, n}=\sum_{i=1}^{n}[(k-1) n+i]^{\beta}$. We have

$$
\left\|D_{n} \xi\right\|_{r, q}^{r}=\sum_{k=1}^{\infty}\left(\xi_{k}^{*}\right)^{r} \sum_{i=1}^{n}[(k-1) n+i]^{\beta}=\sum_{k=1}^{\infty}\left(\xi_{k}^{*}\right)^{r} \lambda_{k, n}
$$

(1) Let $r<q$, so that $-1<\beta<0$. Then

$$
k^{\beta} n^{1+\beta}=n(k n)^{\beta} \leqslant \lambda_{k, n} \leqslant \sum_{i=1}^{n}(k i)^{\beta}=k^{\beta} \sum_{i=1}^{n} i^{\beta} \leqslant k^{\beta}\left(1+\int_{1}^{n} x^{\beta} \mathrm{d} x\right)=\frac{n^{1+\beta}}{1+\beta} k^{\beta} .
$$

Therefore,

$$
n^{1+\beta}\left(\|\xi\|_{r, q}\right)^{r} \leqslant\left(\left\|D_{n} \xi\right\|_{r, q}\right)^{r} \leqslant \frac{n^{1+\beta}}{1+\beta}\left(\|\xi\|_{r, q}\right)^{r}
$$

Thus

$$
\left\|D_{n}\right\| \leqslant\left(\frac{n^{1+\beta}}{1+\beta}\right)^{1 / r}
$$

and, by (3.4), $n^{(1+\beta) / r} \leqslant\left\|D_{1 / n}\right\|^{-1}$. Using (3.5), we have

$$
q \leqslant \lim _{n \rightarrow \infty}\left(\frac{\ln (n)}{\ln \left\|D_{n}\right\|}\right)=p_{\phi} \leqslant q_{\phi}=\lim _{n \rightarrow \infty}\left(\frac{\ln (1 / n)}{\ln \left\|D_{1 / n}\right\|}\right) \leqslant q .
$$

(2) If $r=q$, then $\mathfrak{S}^{q, q}=\mathfrak{S}^{q}$. By (3.6), $\left\|D_{n}\right\|=n^{1 / q}$, so $p_{\phi}=q_{\phi}=q$. 
(3) Let $r>q$, so $0<\beta$. Then

$$
\frac{n^{1+\beta}}{1+\beta} k^{\beta} \leqslant \lambda_{k, n} \leqslant n^{1+\beta} k^{\beta}
$$

since

$$
\frac{n^{1+\beta}}{1+\beta}\left[k^{1+\beta}-(k-1)^{1+\beta}\right]=\int_{0}^{n}[(k-1) n+x]^{\beta} \mathrm{d} x \leqslant \lambda_{k, n} \leqslant n(k n)^{\beta}=n^{1+\beta} k^{\beta} .
$$

Repeating the argument of part (1), we obtain that $p_{\phi}=q_{\phi}=q$.

For $p \in(1, \infty)$, set

$$
p_{-}=\min \left(p, p^{\prime}\right), \quad p_{+}=\max \left(p, p^{\prime}\right), \quad \text { where } \frac{1}{p}+\frac{1}{p^{\prime}}=1 .
$$

Corollary 3.6. Let $J$ be a separable s.n. ideal. If $p_{-}<p_{J}$ and $q_{J}<p_{+}$, then each commutator $\mathfrak{S}^{p}$-bounded function $g$ on $\alpha \subset \mathbb{C}$ is commutator $J$-bounded on $\alpha$. In particular, $g$ is commutator $\mathfrak{S}^{q}$ and $\mathfrak{S}^{r, q}$-bounded on $\alpha$ for $p_{-}<q<p_{+}$and $1 \leqslant r<\infty$.

Proof. Let $p<p^{\prime}$. By Corollary 3.4 of $[\mathbf{2}], J$ is an interpolation space for $\left(\mathfrak{S}^{p}, \mathfrak{S}^{p^{\prime}}\right)$. Since $\phi_{p^{\prime}}=\left(\phi_{p}\right)^{*}$ and $\mathfrak{S}^{p} \subset \mathfrak{S}^{p^{\prime}}$, the result follows from Theorem 3.3.

Recall that $\alpha \subset \mathbb{C}$ is $J$-Fuglede if the function $h(z)=\bar{z}$ is commutator $J$-bounded on $\alpha$, that is, (1.4) holds.

Definition 3.7. An s.n. ideal $J$ is called Fuglede if there is $C>0$ such that, for all normal $A \in J$ and all $X \in B(H)$,

$$
\left\|A^{*} X-X A^{*}\right\|_{J} \leqslant C\|A X-X A\|_{J}
$$

Evidently, an ideal $J$ is Fuglede if and only if all compact subsets of $\mathbb{C}$ are $J$-Fuglede. This is equivalent to the condition that the unit disc of $\mathbb{C}$ is $J$-Fuglede. If $J$ is Fuglede, it follows from Proposition 4.5 of [17] that the spaces of $J$-Lipschitz and of commutator $J$-bounded functions coincide on each compact in $\mathbb{C}$. It was proved in $[\mathbf{1 , 2 4 , 2 5}]$ that all Schatten ideals $\mathfrak{S}^{p}, 1<p<\infty$, are Fuglede. It follows from the results of [15] that $\mathfrak{S}^{b}=B(H)$ is not Fuglede. From this and from Corollary 5.4 of $[\mathbf{1 7}]$ and Corollary 2.5 we obtain the following result.

\section{Corollary 3.8.}

(i) Let $\phi^{*}$ be the adjoint of $\phi$. If one of the ideals $J^{\phi}, J^{\phi^{*}}, J_{0}^{\phi}, J_{0}^{\phi^{*}}$ is Fuglede, then the others are also Fuglede ideals.

(ii) If $1<p_{J}$ and $q_{J}<\infty$, for a separable ideal $J$, then $J$ is a Fuglede ideal. In particular, all Lorentz ideals $\mathfrak{S}^{r, q}$ with $q>1$ are Fuglede ideals.

(iii) The ideals $\mathfrak{S}^{1}, \mathfrak{S}^{\infty}$ and $\mathfrak{S}^{b}$ are not Fuglede ideals. 
Problem 3.9. Are the Lorentz ideals $\mathfrak{S}^{r, 1}$ Fuglede ideals if $1<r$ ?

We now return to the subject of $J$-Lipschitz functions. Combining the results of Proposition 4.5 of [17], of Corollaries 3.6 and 5.4 of [17], and of Corollaries 2.3 and 2.5 yields the following corollary.

Corollary 3.10. Let $g$ be a $\mathfrak{S}^{p}$-Lipschitz function on $\alpha \subset \mathbb{C}$.

(i) Let $p \in(1, \infty)$. If $p_{-}<p_{J}$ and $q_{J}<p_{+}$, for a separable ideal $J=J_{0}^{\phi}$, then $g$ is a $J_{0}^{\phi}-, J_{0}^{\phi^{*}}-, J^{\phi}-$ and $J^{\phi^{*}}$-Lipschitz function on $\alpha$. In particular, $g$ is $\mathfrak{S}^{r, q}$-Lipschitzian on $\alpha$ for $p_{-}<q<p_{+}$(see Proposition 3.5).

(ii) Let $p \in\{1, \infty, b\}$ and let $\alpha$ be a $\mathfrak{S}^{p}$-Fuglede set. Then $g$ is $J_{0}^{\phi}$ - and $J^{\phi}$-Lipschitzian on $\alpha$ for any $\phi$.

Let $J$ - $\operatorname{Lip}(a, b)$ be the space of all $J$-Lipschitz functions on $[a, b]$. By Corollary 3.6 of [17], functions in $\mathfrak{S}^{b}-\operatorname{Lip}(a, b)$ are differentiable. For $p \in(1, \infty)$, the spaces $\mathfrak{S}^{p}-\operatorname{Lip}(a, b)$ are larger than $\mathfrak{S}^{b}-\operatorname{Lip}(a, b)$ and contain Lipschitz, non-differentiable functions (see $[\mathbf{9}]$ ). Peller [22] showed that $B_{\infty 1}^{1}(a, b) \subseteq \mathfrak{S}^{b}-\operatorname{Lip}(a, b) \subseteq B_{11}^{1}(a, b)$, where $B_{\infty 1}^{1}(a, b)$ and $B_{11}^{1}(a, b)$ are Besov classes of functions on $[a, b]$ (for another proof and some interesting related results, see [7]). Combining this with Proposition 5.5 (ii) of [17] and with Corollary 2.9 (ii) yields the following corollary.

Corollary 3.11. For any s.n. function $\phi$,

$$
\begin{aligned}
B_{\infty 1}^{1}(a, b) & \subseteq \mathfrak{S}^{b}-\operatorname{Lip}(a, b)=\mathfrak{S}^{1}-\operatorname{Lip}(a, b) \subseteq J^{\phi}-\operatorname{Lip}(a, b)=J_{0}^{\phi}-\operatorname{Lip}(a, b) \\
& =J^{\phi^{*}}-\operatorname{Lip}(a, b)=J_{0}^{\phi^{*}}-\operatorname{Lip}(a, b) \subseteq \mathfrak{S}^{2}-\operatorname{Lip}(a, b)=\operatorname{Lip}(a, b) .
\end{aligned}
$$

\section{Extension of domains of inequalities (1.2) and (1.3)}

The normal operators in the definitions of $J$-Lipschitz and of commutator $J$-bounded functions (see (1.2) and (1.3)) belong to the ideal $J$. The question arises as to whether inequalities (1.2) and (1.3) hold for all normal operators in $B(H)$. More precisely, we study for which s.n. ideals $J$ the following properties hold.

(i) If $g$ is $J$-Lipschitzian on $\alpha$, then there is $\mathcal{D}>0$ such that, for all normal operators $A, B$ with spectrum in $\alpha$, the condition $A-B \in J$ implies

$$
g(A)-g(B) \in J \quad \text { and } \quad\|g(A)-g(B)\|_{J} \leqslant \mathcal{D}\|A-B\|_{J} .
$$

(ii) If $g$ is commutator $J$-bounded on $\alpha$, then there is $\mathcal{D}>0$ such that, for any normal $A$ with $\operatorname{Sp}(A) \subseteq \alpha$ and any $X$ in $B(H)$, the condition $[A, X] \in J$ implies

$$
[g(A), X] \in J \quad \text { and } \quad\|[g(A), X]\|_{J} \leqslant \mathcal{D}\|[A, X]\|_{J} .
$$

It will be shown that (i) and (ii) hold for all ideals $J^{\phi}$ and $J_{0}^{\phi}$. This extends the result of Kittaneh [18] (see also Jocic [14]), who considered the case $J=\mathfrak{S}^{2}$. 
Let $J=J_{0}^{\phi}$. For $A \in B(H)$, the operator $\delta_{A}: X \rightarrow[A, X]$ is bounded on $J$. If $T \in J^{\phi^{*}}$, it follows from (2.1) that, for $X \in J$,

$$
F_{T}\left(\delta_{A}(X)\right)=\operatorname{Tr}(A X T)-\operatorname{Tr}(X A T)=\operatorname{Tr}(X T A)-\operatorname{Tr}(X A T)=F_{-\delta_{A}(T)}(X) .
$$

Let $L$ be a $*$-subspace of $J: X \in L$ implies $X^{*} \in L$. Denote by $L^{\perp}$ its annihilator in $J^{\phi^{*}}$ :

$$
L^{\perp}=\left\{T \in J^{\phi^{*}}: F_{T}(X)=\operatorname{Tr}(X T)=0 \text { for } X \in L\right\} .
$$

If $T \in L^{\perp}$, then

$$
F_{T^{*}}(X)=\operatorname{Tr}\left(T^{*} X\right)=\operatorname{Tr}\left(\left(X^{*} T\right)^{*}\right)=\overline{\operatorname{Tr}\left(X^{*} T\right)}=\overline{F_{T}\left(X^{*}\right)}=0,
$$

for $X \in L$. Therefore, $T^{*} \in L^{\perp}$, so $L^{\perp}$ is a $*$-subspace of $J^{\phi^{*}}$.

Let $A$ be a normal operator and $\{A\}^{\prime}$ be its commutant. Let $\left\{\mu_{i}\right\}_{i \in I}$ be the set of all eigenvalues of $A$ and let $Q_{i}$ be the projections on the corresponding eigenspaces. Then

$$
B(H) \ni X \rightarrow \Psi_{A}(X)=\sum_{i \in I} Q_{i} X Q_{i}
$$

is a map from $B(H)$ into $\{A\}^{\prime}$ and $\left(\Psi_{A}\right)^{2}=\Psi_{A}$. It follows from Theorem III.4.2 of [12] that $\Psi_{A}(X) \in J$, for $X \in J$, and $\left\|\Psi_{A}(X)\right\|_{J} \leqslant\|X\|_{J}$. Hence $\Psi_{A}$ maps $J$ into $\{A\}^{\prime} \cap J$ and $\left\|\Psi_{A}\right\| \leqslant 1$. By $\overline{\delta_{A}(J)}$ we denote the closure of $\delta_{A}(J)$ in $\|\cdot\|_{J}$.

We will now generalize Theorem 2.2 of $[\mathbf{1 6}]$ to all separable s.n. ideals.

Theorem 4.1. Let $J=J_{0}^{\phi} \neq \mathfrak{S}^{1}$ and let $A$ be a normal operator in $B(H)$. Then

(i) $\operatorname{Ker}\left(\delta_{A}\right)=\{A\}^{\prime} \cap J=\left\{X \in J: \Psi_{A}(X)=X\right\}$ so $\Psi_{A}$ is a projection on $\operatorname{Ker}\left(\delta_{A}\right)$;

(ii) $\operatorname{Ker}\left(\Psi_{A}\right)=\overline{\delta_{A}(J)}$ and $J$ is the direct sum of $\overline{\delta_{A}(J)}$ and $\operatorname{Ker}\left(\delta_{A}\right)$.

Proof. Since $\Psi_{A}$ maps $J$ to $\{A\}^{\prime} \cap J$, we have $\left\{X \in J: \Psi_{A}(X)=X\right\} \subseteq\{A\}^{\prime} \cap J$.

Conversely, let $X=X^{*} \in\{A\}^{\prime} \cap J$. Then $X=\bigoplus_{j=1}^{\infty} \lambda_{j} P_{j}$, where $P_{j}$ are the mutually orthogonal projections on the finite-dimensional eigenspaces $L_{j}$ of $X$. All $P_{j} \in\{A\}^{\prime} \cap J$. Fix $j$. The subspace $L_{j}$ is invariant for $A$ and, therefore, decomposes in the orthogonal sum of eigenspaces of the operator $\left.A\right|_{L_{j}}$. Hence $P_{j}=\Psi_{A}\left(P_{j}\right)$.

The finite sums $X_{n}=\bigoplus_{j=1}^{n} \lambda_{j} P_{j}$ converge to $X$ in $\|\cdot\|_{J}$. Since $\left\|\Psi_{A}\right\| \leqslant 1$,

$$
\begin{aligned}
\left\|X-\Psi_{A}(X)\right\|_{J} & \leqslant\left\|X-X_{n}\right\|_{J}+\left\|\Psi_{A}(X)-X_{n}\right\|_{J} \\
& =\left\|X-X_{n}\right\|_{J}+\left\|\Psi_{A}\left(X-\bigoplus_{j=1}^{n} \lambda_{j} P_{j}\right)\right\|_{J} \leqslant 2\left\|X-X_{n}\right\|_{J} \rightarrow 0 .
\end{aligned}
$$

Hence $\Psi_{A}(X)=X$. By Fuglede's theorem, $\{A\}^{\prime} \cap J$ is a $*$-algebra. Thus $\{A\}^{\prime} \cap J \subseteq\{X \in$ $\left.J: \Psi_{A}(X)=X\right\}$ and part (i) is proved.

Let us show that $L=\delta_{A}(J)+\left(\{A\}^{\prime} \cap J\right)$ is dense in $J$. We have

$$
L^{\perp}=\delta_{A}(J)^{\perp} \cap\left(\{A\}^{\prime} \cap J\right)^{\perp} .
$$


Since $\{A\}^{\prime} \cap J$ is a $*$-subspace, $\left(\{A\}^{\prime} \cap J\right)^{\perp}$ is a $*$-subspace of $J^{\phi^{*}}$. If $T \in \delta_{A}(J)^{\perp}$, it follows from (4.1) that $\delta_{A}(T)=0$. Hence $T \in\{A\}^{\prime} \cap J^{\phi^{*}}$, so $\left(\delta_{A}(J)\right)^{\perp}=\{A\}^{\prime} \cap J^{\phi^{*}}$ is a *-algebra. Thus $L^{\perp}$ is a $*$-subspace of $\{A\}^{\prime} \cap J^{\phi^{*}}$.

Let $T=T^{*} \in L^{\perp} \subseteq J^{\phi^{*}}$. It is compact, so $T=\bigoplus_{i=1}^{\infty} \lambda_{i} P_{i}$, where the $P_{i}$ are mutually orthogonal finite-dimensional projections. Since $T \in\{A\}^{\prime}$, all $P_{i}$ belong to $\{A\}^{\prime} \cap J \subseteq L$. Hence $0=F_{T}\left(P_{i}\right)=\operatorname{Tr}\left(P_{i} T\right)=\lambda_{i} \operatorname{dim}\left(P_{i}\right)$. Thus $T=0$. Since $L^{\perp}$ is a $*$-space, $L^{\perp}=\{0\}$, so $L$ is dense in $J$.

We now proceed with the proof of (ii). Since $\Psi_{A}$ is a projection on $\{A\}^{\prime} \cap J$,

$$
J=\operatorname{Ker}\left(\Psi_{A}\right) \dot{+}\left(\{A\}^{\prime} \cap J\right) .
$$

Taking into account that $Q_{i} A=A Q_{i}=\mu_{i} Q_{i}$, for all $Q_{i}$, we obtain that

$$
\Psi_{A}\left(\delta_{A}(X)\right)=\sum_{i \in I}\left(Q_{i} A X Q_{i}-Q_{i} X A Q_{i}\right)=\sum_{i \in I}\left(\mu_{i} Q_{i} X Q_{i}-\mu_{i} Q_{i} X Q_{i}\right)=0
$$

for any $X \in J$. Since $\left\|\Psi_{A}\right\| \leqslant 1$, we conclude that $\overline{\delta_{A}(J)} \subseteq \operatorname{Ker}\left(\Psi_{A}\right)$.

Let $X \in \operatorname{Ker}\left(\Psi_{A}\right)$. Since $L$ is dense in $J$, there are $X_{n} \in \delta_{A}(J)$ and $Y_{n} \in\{A\}^{\prime} \cap J$ such that $\left\|X-X_{n}-Y_{n}\right\|_{J} \rightarrow 0$. Since $\Psi_{A}\left(Y_{n}\right)=Y_{n}$ and $\Psi_{A}\left(X_{n}\right)=0$, it follows that

$$
\left\|Y_{n}\right\|_{J}=\left\|\Psi_{A}\left(Y_{n}\right)\right\|_{J}=\left\|\Psi_{A}\left(X-X_{n}-Y_{n}\right)\right\|_{J} \leqslant\left\|X-X_{n}-Y_{n}\right\|_{J} \rightarrow 0 .
$$

Hence $\left\|X-X_{n}\right\|_{J} \rightarrow 0$, so $\operatorname{Ker}\left(\Psi_{A}\right)=\overline{\delta_{A}(J)}$ and part (ii) follows from (4.2).

Theorem 1 of $[\mathbf{2 4}]$ is a special case of the following result.

Proposition 4.2. Let $\mathfrak{Y}$ be a linear manifold in a Banach space $\mathfrak{X}$ and a Banach space with norm $\|\cdot\|_{\mathfrak{Y}}$. Let $S$ and $T$ be commuting operators on $\mathfrak{X}$. Assume that

(i) $\operatorname{Ker}(S) \subseteq \operatorname{Ker}(T)$;

(ii) $\operatorname{Ker}(T) \cap \overline{T \mathfrak{X}}=\{0\}$, where $\overline{T \mathfrak{X}}$ is the norm closure of $T \mathfrak{X}$;

(iii) $S$ and $T$ preserve $\mathfrak{Y}$ and the operators $\left.S\right|_{\mathfrak{Y}}$ and $\left.T\right|_{\mathfrak{Y}}$ are bounded on $\mathfrak{Y}$;

(iv) $\|y\|_{\mathfrak{X}} \leqslant\|y\|_{\mathfrak{Y}}$ and $\|T y\|_{\mathfrak{Y}} \leqslant\|S y\|_{\mathfrak{Y}}$, for $y \in \mathfrak{Y}$;

(v) $\mathfrak{Y}$ is the direct sum of $\operatorname{Ker}\left(\left.S\right|_{\mathfrak{Y}}\right)$ and the closure $\overline{S \mathfrak{Y}}$ of $S \mathfrak{Y}$ in $\|\cdot\|_{\mathfrak{Y}}$.

Let $P$ be the projection in $\mathfrak{Y}$ on $\operatorname{Ker}\left(\left.S\right|_{\mathfrak{Y}}\right)$ along $\overline{S \mathfrak{Y}}$. Then

$S x \in \mathfrak{Y}, \quad$ for $x \in \mathfrak{X}, \quad$ implies $T x \in \mathfrak{Y}$ and $\|T x\|_{\mathfrak{Y}} \leqslant(1+\|P\|)\|S x\|_{\mathfrak{Y}}$.

Proof. Define an operator $U$ on $\operatorname{Ker}\left(\left.S\right|_{\mathfrak{Y}}\right) \dot{+} S \mathfrak{Y}$ by the formula

$$
U z=0, \quad \text { for } z \in \operatorname{Ker}\left(\left.S\right|_{\mathfrak{Y}}\right), \quad \text { and } \quad U S y=T y, \quad \text { for } y \in \mathfrak{Y} .
$$

By (iv),

$\|U(z+S y)\|_{\mathfrak{Y}}=\|T y\|_{\mathfrak{Y}} \leqslant\|S y\|_{\mathfrak{Y}} \leqslant\|z+S y\|_{\mathfrak{Y}}+\|P(z+S y)\|_{\mathfrak{Y}} \leqslant(1+\|P\|)\|z+S y\|_{\mathfrak{Y}}$. 
Hence $U$ extends to a bounded operator on $\mathfrak{Y}$ and $\|U\| \leqslant 1+\|P\|$. Since $S, T$ commute and $\operatorname{Ker}(S) \subseteq \operatorname{Ker}(T)$, we have $S U(z+S y)=S T y=T S y=T(z+S y)$. Thus

$$
\left.T\right|_{\mathfrak{Y}}=\left.U S\right|_{\mathfrak{Y}}=\left.S U\right|_{\mathfrak{Y}} .
$$

Let $x \in \mathfrak{X}$ and $S x \in \mathfrak{Y}$. Then $S(T x-U S x)=S T x-S U S x=S T x-T S x=0$. Hence $T x-U S x \in \operatorname{Ker}(S)$. By (i), $T x-U S x \in \operatorname{Ker}(T)$. Since $S x \in \mathfrak{Y}$, there are $z \in \operatorname{Ker}\left(\left.S\right|_{\mathfrak{Y}}\right)$ and $y_{n} \in \mathfrak{Y}$ such that $\left\|S x-\left(z+S y_{n}\right)\right\|_{\mathfrak{Y}} \rightarrow 0$. Therefore,

$\left\|U S x-T y_{n}\right\|_{\mathfrak{X}} \leqslant\left\|U S x-T y_{n}\right\|_{\mathfrak{Y}}=\left\|U S x-U\left(z+S y_{n}\right)\right\|_{\mathfrak{Y}} \leqslant\|U\|\left\|S x-\left(z+S y_{n}\right)\right\|_{\mathfrak{Y}} \rightarrow 0$.

Thus $U S x \in \overline{T \mathfrak{X}}$, so $T x-U S x \in \operatorname{Ker}(T) \cap \overline{T \mathfrak{X}}$. It follows from (ii) that $T x=U S x \in \mathfrak{Y}$ and $\|T x\|_{\mathfrak{Y}} \leqslant\|U\|\|S x\|_{\mathfrak{Y}} \leqslant(1+\|P\|)\|S x\|_{\mathfrak{Y}}$.

A bounded operator $T$ on a Banach space $\mathfrak{X}$ is called hermitian, if $\|\exp (\mathrm{i} t T)\|_{\mathfrak{X}}=1$ for $t \in \mathbb{R}$. It is called normal if $T=A+\mathrm{i} B$, where $A$ and $B$ are commuting hermitian operators. Fong $[\mathbf{1 1}]$ showed that if $T$ is normal, then

$$
\operatorname{Ker}(T) \cap \overline{T \mathfrak{X}}=\{0\} .
$$

Let $K$ be a self-adjoint operator in $B(H)$. The operator $\delta_{K}(X)=K X-X K$ is hermitian on any s.n. ideal $J$, since

$$
\left\|\exp \left(\mathrm{i} t \delta_{K}\right) X\right\|_{J}=\left\|\mathrm{e}^{\mathrm{i} t K} X \mathrm{e}^{-\mathrm{i} t K}\right\|_{J}=\|X\|_{J} \quad \text { for } X \in J .
$$

If $S$ is a normal operator on $H, S=K+\mathrm{i} L$, where the operators $K$ and $L$ are self-adjoint and commute. Then the operators $\delta_{K}$ and $\delta_{L}$ on $J$ are hermitian and commute, so the operator $\delta_{S}=\delta_{K}+\mathrm{i} \delta_{L}$ is normal on $J$. We will show now that 'extended' inequalities (1.2) and (1.3) hold for all separable s.n. ideals.

Theorem 4.3. Let $J$ be a separable s.n. ideal and let $g$ be a commutator $J$-bounded function on $\alpha \subset \mathbb{C}$. There is $\mathcal{D}>0$ such that, for $X \in B(H)$ and normal operators $A, B$ with spectra in $\alpha, A X-X B \in J$ implies

$$
g(A) X-X g(B) \in J \quad \text { and } \quad\|g(A) X-X g(B)\|_{J} \leqslant \mathcal{D}\|A X-X B\|_{J} .
$$

Proof. Since $g$ is commutator $J$-bounded, it follows from Proposition 3.4 (iii) of [17] that there is $D>0$ such that, for all normal $A$ with $\operatorname{Sp}(A) \subseteq \alpha$ and all $X \in J$,

$$
\left\|\delta_{g(A)}(X)\right\|_{J}=\|[g(A), X]\|_{J} \leqslant D\|[A, X]\|_{J}=D\left\|\delta_{A}(X)\right\|_{J}
$$

Let $J \neq \mathfrak{S}^{1}$. The operator $g(A)$ is normal, so that the operators $\delta_{A}, \delta_{g(A)}$ are normal on $J$ and on $B(H)$, commute and $\operatorname{Ker}\left(\delta_{A}\right) \subseteq \operatorname{Ker}\left(\delta_{g(A)}\right)$. It follows from Theorem 4.1 that $J=\operatorname{Ker}\left(\left.\delta_{A}\right|_{J}\right) \dot{+} \overline{\delta_{A}(J)}$ and the projection on $\operatorname{Ker}\left(\left.\delta_{A}\right|_{J}\right)$ has norm 1. By (4.3), $\operatorname{Ker}\left(\delta_{g(A)}\right) \cap \overline{\delta_{g(A)}(B(H))}=\{0\}$. Replacing $T$ in Proposition 4.2 by $\delta_{g(A)}, S$ by $\delta_{A}, \mathfrak{X}$ by $B(H)$ and $\mathfrak{Y}$ by $J$, we obtain that, for any $X \in B(H)$,

$$
[A, X] \in J \quad \text { implies }[g(A), X] \in J \text { and }\|[g(A), X]\|_{J} \leqslant 2 D\|[A, X]\|_{J} .
$$

Repeating now the argument of Proposition 4.1 of [17], we complete the proof of the case $J \neq \mathfrak{S}^{1}$. The case when $J=\mathfrak{S}^{1}$ will be considered in Theorem 4.5. 
To prove an analogue of Theorem 4.3 for all ideals $J^{\phi}$, we need the following result.

Lemma 4.4. Let $J \subset I \subseteq C(H)$ and let $J=J^{\phi}$. Then there are s.n. ideals $\{J(t)\}_{t \in[0,1]}$ such that

(i) $J=J(1) \subseteq J(t) \subseteq J(s) \subseteq J(0)=I$, for $s \leqslant t$ in $(0,1)$;

(ii) all functions $\varphi_{X}(t)=\|X\|_{J(t)}$ are continuous on $[0,1]$ for $X \in J$;

(iii) all $J(t)$ are interpolation spaces for the pair $(J, I)$ and if $T$ is a bounded operator on $(J, I)$, then $\|T\|_{J(t)} \leqslant\|T\|_{I}^{t}\|T\|_{J}^{1-t}$ for $t \in[0,1]$;

(iv) if $I$ is reflexive, then all ideals $J(t), t \in(0,1)$, are reflexive.

Proof. It follows from Theorem III.5.1 of [12] that the unit ball $\mathbf{J}_{1}$ of $J$ is closed in $(C(H),\|\cdot\|)$. By Proposition 2.1, there is $c>0$ such that $\|X\| \leqslant c\|X\|_{I}$ for $X \in I$. This implies that $\mathbf{J}_{1}$ is closed in $I$ in $\|\cdot\|_{I}$.

By Proposition 2.1 (i), there is $C>0$ such that $\|X\|_{I} \leqslant C\|X\|_{J}$, for $X \in J$. Consider an equivalent norm $\|X\|_{J}^{\prime}=C\|X\|_{J}$ on $J$. Then $\|X\|_{I} \leqslant\|X\|_{J}^{\prime}$. It follows from Theorems IV.1.2 and IV.1.8 of [19] that there are Banach spaces $\left(J(t),\|\cdot\|_{J(t)}\right)$, for $t \in[0,1]$, which satisfy (i), (ii) and (iii) and such that $\|X\|_{J(s)} \leqslant\|X\|_{J(t)}$, for $s \leqslant t$ and $X \in J(t)$. By Lemma 3.2 , all $\left(J(t),\|\cdot\|_{J(t)}\right)$ are s.n. ideals.

If $I$ is reflexive, it follows from Theorem IV.1.4 of [19] that all s.n. ideals $J(t), t \in(0,1)$, are also reflexive.

All compact operators $B$ such that $\sum_{n=1}^{\infty} n^{-1 / 2} s_{n}(B)<\infty$, where $s_{n}(B)$ are eigenvalues of $\left(B^{*} B\right)^{1 / 2}$, form a separable ideal $\mathfrak{S}_{-}^{2}$ (see $\left.[\mathbf{1 2}, \S 15]\right)$ contained in $\mathfrak{S}^{2}$. Let $J$ be an s.n. ideal not contained in $\mathfrak{S}_{-}^{2}$. Bercovici and Voiculescu proved in $[\mathbf{3}]$ that, for any normal $A$ in $B(H)$, there are finite-rank positive operators $R_{n}$ such that

the $R_{n}$ strongly converge to $1, \quad\left\|R_{n}\right\| \leqslant 1 \quad$ and $\quad \lim \left\|\left[A, R_{n}\right]\right\|_{J}=0$.

Theorem 4.5. Let $J=J^{\phi}$. If $g$ is commutator $J$-bounded on $\alpha \subset \mathbb{C}$, then there is $\mathcal{D}>0$ such that, for all normal operators $A, B$ with spectra in $\alpha$ and all $X \in B(H)$,

$A X-X B \in J \quad$ implies $g(A) X-X g(B) \in J$ and $\|g(A) X-X g(B)\|_{J} \leqslant \mathcal{D}\|A X-X B\|_{J}$.

Proof. We will prove the theorem for $A=B$. Then the general case for different $A$ and $B$ will follow as in the proof of Proposition 4.1 of [17].

(1) Let $J \nsubseteq \mathfrak{S}_{-}^{2}$. By Proposition 3.4 of $[\mathbf{1 7}]$, there is $D>0$ such that $\|[g(A), Y]\|_{J} \leqslant$ $D\|[A, Y]\|_{J}$ for all normal $A$ with $\operatorname{Sp}(A) \subseteq \alpha$ and all $Y \in J$. Fix $A$. Let finite-rank operators $R_{n}$ satisfy (4.5). Then, for all $X \in B(H)$,

$$
\left\|\left[g(A), R_{n}\right]\right\|_{J} \leqslant D\left\|\left[A, R_{n}\right]\right\|_{J} \rightarrow 0 \quad \text { and } \quad\left\|\left[g(A), R_{n} X\right]\right\|_{J} \leqslant D\left\|\left[A, R_{n} X\right]\right\|_{J} .
$$

Taking this into account, we have from Lemma 3.3 of $[\mathbf{1 7}]$

$$
\begin{aligned}
\|[g(A), X]\|_{J} & =\lim \left\|R_{n}[g(A), X]\right\|_{J}=\lim \left\|\left[g(A), R_{n} X\right]-\left[g(A), R_{n}\right] X\right\|_{J} \\
& \leqslant D \lim \left\|\left[A, R_{n} X\right]\right\|_{J} \leqslant D \lim \left\|\left[A, R_{n}\right] X\right\|_{J}+D \lim \left\|R_{n}[A, X]\right\|_{J} \\
& =D\|[A, X]\|_{J}
\end{aligned}
$$


(2) Let $J \subseteq \mathfrak{S}_{-}^{2}$. By (1.3), there is $D>0$ such that $\|[g(A), X]\|_{J} \leqslant D\|[A, X]\|_{J}$ for all $A \in J_{\text {nor }}(\alpha)$ and $X \in B(H)$. Since $A$ are diagonal, by Proposition 5.2 of [17], the matrices $M(A, g)$ (see $\S 3)$ are Hadamard $J$-multipliers and $\|M(A, g)\|_{J} \leqslant 2 D$. Since $g$ is a Lipschitz function, we have from (5.1) and (5.2) of [17] that $M(A, g)$ are also Hadamard $\mathfrak{S}^{2}$-multipliers and $\|M(A, g)\|_{\mathfrak{S}^{2}} \leqslant\|M(A, g)\|_{J}$.

Since $J \subset \mathfrak{S}^{2}$ and $\mathfrak{S}^{2}$ is reflexive, there are s.n. ideals $J(t), t \in[0,1]$, which satisfy conditions of Lemma 4.4: $J=J(1), \mathfrak{S}^{2}=J(0)$ and $J(t)$ are reflexive for $t \in(0,1)$. By Lemma 4.4 (iii), the $M(A, g)$ are also Hadamard $J(t)$-multipliers, for $t \in(0,1)$, and $\|M(A, g)\|_{J(t)} \leqslant 2 D$. It follows from Proposition 5.2 of $[\mathbf{1 7}]$ that

$$
\|[g(A), X]\|_{J(t)} \leqslant 2 D\|[A, X]\|_{J(t)} \quad \text { for } A \in J_{\text {nor }}(\alpha) \text { and } X \in J(t) .
$$

By Proposition 2.1 (iii), all s.n. ideals $J(t), t \in(0,1)$, are separable. It follows from Proposition 3.4 of $[\mathbf{1 7}]$ that $g$ is commutator $J(t)$-bounded and (4.6) holds for all normal operators $A$ with $\operatorname{Sp}(A) \subseteq \alpha$ and all $X \in J(t)$. Since all $J(t)$ are reflexive, $J(t) \neq \mathfrak{S}^{1}$. It was proved in Theorem 4.3 that $[A, X] \in J(t)$ implies

$$
[g(A), X] \in J(t) \quad \text { and } \quad\|[g(A), X]\|_{J(t)} \leqslant 4 D\|[A, X]\|_{J(t)},
$$

for $t \in(0,1)$ and for all normal $A$ with $\operatorname{Sp}(A) \subseteq \alpha$ and all $X \in B(H)$.

If $[A, X] \in J$, then $[A, X] \in J(t)$, for $t \in(0,1)$, so (4.7) holds. By (1.1) and (4.7), $\|[g(A), X] P\|_{J(t)} \leqslant 4 D\|[A, X]\|_{J(t)}$ for any finite-dimensional projection $P$. It follows from Lemma 4.4 (ii) that

$$
\|[g(A), X] P\|_{J}=\lim _{t \rightarrow 1}\|[g(A), X] P\|_{J(t)} \leqslant 4 D \lim _{t \rightarrow 1}\|[A, X]\|_{J(t)}=4 D\|[A, X]\|_{J} .
$$

Hence, by Theorem III.5.1 of $[\mathbf{1 2}],[g(A), X] \in J$ and $\|[g(A), X]\|_{J} \leqslant 4 D\|[A, X]\|_{J}$.

This completes the proof of the theorem in the case when $A=B$.

To prove the results of Theorems 4.3 and 4.5 for $J$-Lipschitz functions we, as usual, have to restrict our consideration either to Fuglede ideals or to $J$-Fuglede sets.

Corollary 4.6. Let $J$ be $J^{\phi}$ or $J_{0}^{\phi}$ and let $g$ be a $J$-Lipschitz function on a $J$-Fuglede set $\alpha$ (for example, $\alpha \subset \mathbb{R}$ ). Then there is $\mathcal{D}>0$ such that, for all normal operators $A, B$ with spectra in $\alpha$ and all $X \in B(H), A X-X B \in J$ implies

$$
g(A) X-X g(B) \in J \quad \text { and } \quad\|g(A) X-X g(B)\|_{J} \leqslant \mathcal{D}\|A X-X B\|_{J} .
$$

In particular, $A-B \in J$ implies $g(A)-g(B) \in J$ and $\|g(A)-g(B)\|_{J} \leqslant \mathcal{D}\|A-B\|_{J}$.

\section{5. $J$-stable and commutator $J$-stable functions}

In this section we study $J$-stable functions.

Definition 5.1. Let $g$ be a function on $\alpha \subset \mathbb{C}$ and let $J$ be an s.n. ideal.

(i) $g$ is called $J$-stable if, for all normal operators $A, B$ with spectra in $\alpha$, the condition $A-B \in J$ implies $g(A)-g(B) \in J$. 
(ii) $g$ is called commutator $J$-stable if, for all normal operators $A$ with $\operatorname{Sp}(A) \subseteq \alpha$ and all $X \in B(H)$, the condition $[A, X] \in J$ implies $[g(A), X] \in J$.

$J$-stable functions on $\mathbb{R}$ may be considered to be acting on $B(H) / J$. We show that in many important cases $\left(J=\mathfrak{S}^{p}, p \in(1, \infty)\right.$, for example) a function is $J$-stable if and only if it is $J$-Lipschitzian.

It follows from Theorems 4.3 and 4.5 that if $J$ is $J^{\phi}$ or $J_{0}^{\phi}$, then commutator $J$-bounded functions are $J$-stable and commutator $J$-stable. If $J$ is Fuglede, then, by Corollary 4.6, $J$-Lipschitz functions are $J$-stable and commutator $J$-stable. We will study the converse inclusion.

The following result establishes an important relation between the classes of $J$-stable and commutator $J$-stable functions. In particular, it shows that all commutator $J$-stable functions are $J$-stable.

Proposition 5.2. Let $J$ be an s.n. ideal and let $g$ be a continuous function on $\alpha \subset \mathbb{C}$. The following conditions are equivalent:

(i) $g$ is $J$-stable on $\alpha$;

(ii) for any normal operator $A$ with $\operatorname{Sp}(A) \subseteq \alpha$ and any $X=X^{*} \in B(H)$, the condition $[A, X] \in J$ implies $[g(A), X] \in J$.

Proof. (i) $\Longrightarrow$ (ii). Let $A$ be normal, $\operatorname{Sp}(A) \subseteq \alpha$ and let $U$ be a unitary operator such that $[A, U] \in J$. Then $A-U A U^{*}=[A, U] U^{*} \in J$ and $\operatorname{Sp}(A)=\operatorname{Sp}\left(U A U^{*}\right)$. If $g$ is $J$-stable, $g(A)-g\left(U A U^{*}\right) \in J$. Since $g\left(U A U^{*}\right)=U g(A) U^{*}$, we have $[g(A), U] \in J$.

Let $X=X^{*},\|X\|<1$ and $[A, X] \in J$. The operator $U=X+\mathrm{i}(\mathbf{1}-X)^{1 / 2}$ is unitary. Since $f(t)=t+\mathrm{i}(1-t)^{1 / 2}$ is an analytic function in a neighbourhood of $\operatorname{Sp}(X)$, it follows from Example 4.2 of $[\mathbf{1 7}]$ that $[A, U]=[A, f(X)] \in J$. By the above argument, $[g(A), U] \in J$. Similarly, $\left[g(A), U^{*}\right] \in J$, so that

$$
[g(A), X]=\frac{1}{2}\left([g(A), U]+\left[g(A), U^{*}\right]\right) \in J .
$$

From this it follows that, for each $X=X^{*},[A, X] \in J$ implies $[g(A), X] \in J$.

(ii) $\Longrightarrow$ (i). Let $A, B$ be normal operators with spectra in $\alpha$ and let $A-B \in J$. The operator

$$
R=\left(\begin{array}{cc}
A & 0 \\
0 & B
\end{array}\right)
$$

is normal and $\operatorname{Sp}(R) \subseteq \alpha$. Set

$$
X=\left(\begin{array}{ll}
0 & 1 \\
1 & 0
\end{array}\right)
$$

Then $X=X^{*}$ and

$$
[R, X]=\left(\begin{array}{cc}
0 & A-B \\
B-A & 0
\end{array}\right) \in J, \quad \text { so }[g(R), X]=\left(\begin{array}{cc}
0 & g(A)-g(B) \\
g(B)-g(A) & 0
\end{array}\right) \in J .
$$

Hence $g(A)-g(B) \in J$. 
Definition 5.3. Let $h(z)=\bar{z}$.

(i) A compact $\alpha$ in $\mathbb{C}$ is called weakly $J$-Fuglede if the function $h$ is commutator $J$ stable on $\alpha$, that is, for all normal $A$ with $\operatorname{Sp}(A) \subseteq \alpha$ and all $X$ in $B(H),[A, X] \in J$ implies $\left[A^{*}, X\right] \in J$.

(ii) An s.n. ideal $J$ is called weakly Fuglede if any compact subset of $\mathbb{C}$ is weakly $J$ Fuglede, that is, for all normal $A$ and all $X \in B(H),[A, X] \in J$ implies $\left[A^{*}, X\right] \in J$.

Clearly, any compact $\alpha$ in $\mathbb{R}$ is weakly $J$-Fuglede for any ideal $J$.

If $J$ is $J^{\phi}$ or $J_{0}^{\phi}$ and $\alpha \subset \mathbb{C}$ is a $J$-Fuglede set, then the function $h$ is commutator $J$-bounded on $\alpha$. It follows from Theorems 4.3 and 4.5 that $h$ is commutator $J$-stable, so $\alpha$ is weakly $J$-Fuglede.

Similarly, if $J$ is a Fuglede ideal, it is also weakly Fuglede. The converse statement, however, is not true, which justifies our terminology.

Proposition 5.4. The ideal $\mathfrak{S}^{\infty}$ is a weakly Fuglede but not Fuglede ideal.

Proof. By Corollary $3.8, \mathfrak{S}^{\infty}$ is not Fuglede. Let $A$ be normal and $[A, X] \in \mathfrak{S}^{\infty}$. Let $\hat{A}$ and $\hat{X}$ be their images in the Calkin algebra $B(H) / \mathfrak{S}^{\infty}$. Then $[\hat{A}, \hat{X}]=0$. By the Fuglede theorem for $C^{*}$-algebras, $\left[\hat{A}^{*}, \hat{X}\right]=0$, so $\left[A^{*}, X\right] \in \mathfrak{S}^{\infty}$.

We conclude from Proposition 5.4 that every compact in $\mathbb{C}$ is weakly $\mathfrak{S}^{\infty}$-Fuglede, but not every compact is $\mathfrak{S}^{\infty}$-Fuglede.

Proposition 5.5. Let $g$ be a continuous function on $\alpha$. If $\alpha$ is weakly $J$-Fuglede, then the following conditions are equivalent:

(i) $g$ is $J$-stable on $\alpha$;

(ii) $g$ is commutator $J$-stable on $\alpha$.

Proof. (ii) $\Longrightarrow$ (i). This follows from Proposition 5.2.

(i) $\Longrightarrow$ (ii). Let $X=Y+\mathrm{i} Z, Y=Y^{*}, Z=Z^{*}$, and let $[A, X] \in J$ for a normal $A$ with $\operatorname{Sp}(A) \subseteq \alpha$. Since $\alpha$ is weakly $J$-Fuglede, $\left[A, X^{*}\right] \in J$. Hence $[A, Y],[A, Z] \in J$ and, by Proposition 5.2, $[g(A), Y] \in J$ and $[g(A), Z] \in J$. Therefore, $[g(A), X] \in J$.

Let $J$ be an s.n. ideal. For any $n$, set

$$
\beta_{J}(n)=\inf _{X \in \mathcal{F}} \frac{\|\overbrace{X \oplus \cdots \oplus X}^{n}\|_{J}}{\|X\|_{J}} \text { and } \gamma_{J}(n)=\sup _{X \in \mathcal{F}} \frac{\|\overbrace{X \oplus \cdots \oplus X}^{n}\|_{J}}{\|X\|_{J}} .
$$

Then $($ see $(3.2)) \beta_{J}^{*}(n) \leqslant \beta_{J}(n) \leqslant \gamma_{J}(n) \leqslant \gamma_{J}^{*}(n)$. If $J$ is separable, then $\beta_{J}^{*}(n)=\beta_{J}(n)$ and $\gamma_{J}(n)=\gamma_{J}^{*}(n)$. We say that $J$ is regular, if

$$
J \varsubsetneqq \mathfrak{S}^{\infty} \text { and } \varliminf_{n \rightarrow \infty} \frac{\beta_{J}(n)}{\gamma_{J}(n)}>0 .
$$

All ideals $\mathfrak{S}^{p}, p \in[1, \infty)$, are regular with $\beta_{\mathfrak{S}^{p}}(n)=\gamma_{\mathfrak{S}^{p}}(n)=n^{1 / p}$. All Lorentz ideals $J=\mathfrak{S}^{p, q}, 1 \leqslant p, q<\infty$, are regular with $C n^{1 / q} \leqslant \beta_{J}(n) \leqslant \gamma_{J}(n) \leqslant D n^{1 / q}$, for some $0<C \leqslant D$. 
Theorem 5.6. Let $J$ be $J_{0}^{\phi}$ or $J^{\phi}$ and let $g$ be a continuous function on $\alpha$. If $J$ is regular, then the following conditions are equivalent:

(i) $g$ is commutator J-bounded on $\alpha$;

(ii) $g$ is commutator $J$-stable on $\alpha$.

Proof. (i) $\Longrightarrow$ (ii). This follows from Theorems 4.3 and 4.5 .

(ii) $\Longrightarrow$ (i). Let $g$ be commutator $J$-stable, but not commutator $J$-bounded. By Proposition 3.4 of $[\mathbf{1 7}]$, there are some $A_{i} \in \mathcal{F}_{\text {nor }}(\alpha)$ and $X_{i} \in \mathcal{F}$ such that $\left\|\left[g\left(A_{i}\right), X_{i}\right]\right\|_{J} \geqslant$ $i^{3}\left\|\left[A_{i}, X_{i}\right]\right\|_{J}$. Set $\lambda_{i}=i^{2} \max \left(\left\|X_{i}\right\|,\left\|\left[A_{i}, X_{i}\right]\right\|_{J}\right)$ and $Z_{i}=X_{i} / \lambda_{i}$. Then

$$
\left\|Z_{i}\right\| \leqslant i^{-2}, \quad\left\|\left[A_{i}, Z_{i}\right]\right\|_{J} \leqslant i^{-2} \text { and }\left\|\left[g\left(A_{i}\right), Z_{i}\right]\right\|_{J} \geqslant i^{3}\left\|\left[A_{i}, Z_{i}\right]\right\|_{J} .
$$

Set

$$
A=\overbrace{A_{1} \oplus \cdots \oplus A_{1}}^{m_{1}} \oplus \cdots \oplus \overbrace{A_{i} \oplus \cdots \oplus A_{i}}^{m_{i}} \oplus \cdots
$$

and

$$
Z=\overbrace{Z_{1} \oplus \cdots \oplus Z_{1}}^{m_{1}} \oplus \cdots \oplus \overbrace{Z_{i} \oplus \cdots \oplus Z_{i}}^{m_{i}} \oplus \cdots
$$

(we will choose $m_{i}$ later). Then $Z \in C(H)$. Since $A_{i} \in \mathcal{F}_{\text {nor }}(\alpha)$, we have that $\left\|A_{i}\right\| \leqslant$ $\sup _{\lambda \in \alpha}|\lambda|$, so that $A$ is a normal bounded operator with $\operatorname{Sp}(A) \subseteq \alpha$. Set

$$
[A, Z]^{(i)}=\overbrace{\left[A_{1}, Z_{1}\right] \oplus \cdots \oplus\left[A_{1}, Z_{1}\right]}^{m_{1}} \oplus \cdots \oplus \overbrace{\left[A_{i}, Z_{i}\right] \oplus \cdots \oplus\left[A_{i}, Z_{i}\right]}^{m_{i}} .
$$

Then $[A, Z]^{(i)} \in J_{0}^{\phi}$ and, by $(5.1)$,

$$
\begin{aligned}
\left\|[A, Z]^{(i+p)}-[A, Z]^{(i)}\right\|_{J} & \leqslant \sum_{k=i+1}^{i+p}\|\overbrace{\left[A_{k}, Z_{k}\right] \oplus \cdots \oplus\left[A_{k}, Z_{k}\right]}^{m_{k}}\|_{J} \\
& \leqslant \sum_{k=i+1}^{i+p} \gamma\left(m_{k}\right)\left\|\left[A_{k}, Z_{k}\right]\right\|_{J} .
\end{aligned}
$$

It follows from (5.2) that $1 \leqslant k^{-2}\left\|\left[A_{k}, Z_{k}\right]\right\|_{J}^{-1}$. Since $\gamma(i) \rightarrow \infty$ and $\gamma(i+1) \leqslant \gamma(i)+1$ (see $[\mathbf{1 2}$, III.§ 3$]$ ), we may choose $m_{k}$ in such a way that

$$
1 \leqslant k^{-2}\left\|\left[A_{k}, Z_{k}\right]\right\|_{J}^{-1} \leqslant \gamma\left(m_{k}\right) \leqslant k^{-2}\left\|\left[A_{k}, Z_{k}\right]\right\|_{J}^{-1} k^{1 / 2} .
$$

Therefore,

$$
k^{-2} \leqslant \gamma\left(m_{k}\right)\left\|\left[A_{k}, Z_{k}\right]\right\|_{J} \leqslant k^{-3 / 2},
$$

so that

$$
\left\|[A, Z]^{(i+p)}-[A, Z]^{(i)}\right\|_{J} \leqslant \sum_{k=i+1}^{i+p} k^{-3 / 2} \rightarrow 0,
$$


as $i \rightarrow \infty$. Hence $[A, Z]^{(i)}$ converge to some $B \in J_{0}^{\phi}$. From (5.2) we have

$$
\left\|[A, Z]-[A, Z]^{(i)}\right\|=\sup _{i+1 \leqslant k}\left\|\left[A_{k}, Z_{k}\right]\right\| \leqslant \sup _{i+1 \leqslant k}\left\|\left[A_{k}, Z_{k}\right]\right\|_{J} \rightarrow 0,
$$

as $i \rightarrow \infty$. Thus $B=[A, Z] \in J_{0}^{\phi} \subseteq J$. Since $g$ is commutator $J$-stable, $[g(A), Z] \in J$.

On the other hand,

$$
\beta\left(m_{k}\right)\left\|\left[g\left(A_{k}\right), Z_{k}\right]\right\|_{J} \leqslant\|\overbrace{\left[g\left(A_{k}\right), Z_{k}\right] \oplus \cdots \oplus\left[g\left(A_{k}\right), Z_{k}\right]}^{m_{k}}\|_{J} \leqslant\|[g(A), Z]\|_{J} .
$$

Hence it follows from (5.2) that

$$
\beta\left(m_{k}\right) k^{3}\left\|\left[A_{k}, Z_{k}\right]\right\|_{J} \leqslant\|[g(A), Z]\|_{J} .
$$

Therefore,

$$
\frac{\beta_{J}\left(m_{k}\right)}{\gamma_{J}\left(m_{k}\right)} k\left(k^{2} \gamma\left(m_{k}\right)\left\|\left[A_{k}, Z_{k}\right]\right\|_{J}\right) \leqslant\|[g(A), Z]\|_{J}
$$

so that, by (5.3),

$$
\frac{\beta_{J}\left(m_{k}\right)}{\gamma_{J}\left(m_{k}\right)} k \leqslant\|[g(A), Z]\|_{J}
$$

Since $J$ is regular,

$$
\frac{\beta_{J}\left(m_{k}\right)}{\gamma_{J}\left(m_{k}\right)} k \rightarrow \infty, \quad \text { as } k \rightarrow \infty
$$

Thus $[g(A), Z] \notin J$. This contradiction completes the proof.

Corollary 5.7. Let $J$ be $J_{0}^{\phi}$ or $J^{\phi}$ and let it be regular. Then

(i) $J$ is a Fuglede ideal if and only if it is weakly Fuglede;

(ii) a compact $\alpha$ in $\mathbb{C}$ is $J$-Fuglede if and only if it is weakly $J$-Fuglede.

Since $\mathfrak{S}^{1}$ is a regular but not Fuglede ideal, we obtain the following result.

Corollary 5.8. The ideal $\mathfrak{S}^{1}$ is not weakly Fuglede.

The result in Corollary 5.8 means that $[A, X] \in \mathfrak{S}^{1}$, for a normal $A$ and bounded $X$, does not always imply $\left[A^{*}, X\right] \in \mathfrak{S}^{1}$. Weiss $[\mathbf{2 5}]$ asked whether for compact $X$ this implication always holds. A negative answer to this question was obtained in $[\mathbf{2 3}]$. It was established later in [16] that there exists a normal operator $A$ such that, for any $p>1$, one can find an operator $X$ in $\mathfrak{S}^{p}$ such that $[A, X] \in \mathfrak{S}^{1}$ and $\left[A^{*}, X\right] \notin \mathfrak{S}^{1}$.

Corollary 5.9. There exist a compact normal operator $A$ and a compact operator $X$ such that $[A, X] \in \mathfrak{S}^{1}$ and $\left[A^{*}, X\right] \notin \mathfrak{S}^{1}$. 
Proof. It follows from the discussion after Proposition 4.10 of [17] and from Corollary 5.4 of $[\mathbf{1 7}]$ that the sets

$$
\alpha_{k}=\left\{ \pm \frac{1}{l}+\frac{\mathrm{i}}{l}: k \leqslant l<\infty\right\}, \quad k=1,2, \ldots,
$$

are not $\mathfrak{S}^{1}$-Fuglede, so the function $h(z)=\bar{z}$ is not commutator $\mathfrak{S}^{1}$-bounded on them. Set $\alpha_{k(1)}=\alpha_{1}$. By Proposition 3.4 of [17], there are $X_{1} \in \mathcal{F}$ and $A_{1} \in \mathcal{F}_{\text {nor }}\left(\alpha_{k(1)}\right)$ such that $\left\|\left[h\left(A_{1}\right), X_{1}\right]\right\|_{\mathfrak{S}^{1}} \geqslant\left\|\left[A_{1}, X_{1}\right]\right\|_{\mathfrak{S}^{1}}$. Let $k(2) \in \mathbb{N}$ be such that $\alpha_{k(2)} \cap \operatorname{Sp}\left(A_{1}\right)=0$. By Proposition 3.4 of $[\mathbf{1 7}]$, there are $A_{2} \in \mathcal{F}_{\text {nor }}\left(\alpha_{k(2)}\right)$ and $X_{2} \in \mathcal{F}$ such that $\left\|\left[h\left(A_{2}\right), X_{2}\right]\right\|_{\mathfrak{S}^{1}} \geqslant 2^{3}\left\|\left[A_{2}, X_{2}\right]\right\|_{\mathfrak{S}^{1}}$. Continuing this process we get a sequence of sets $\alpha_{k(1)} \supset \alpha_{k(2)} \supset \cdots \supset \alpha_{k(l)}$ and sequences of normal operators $A_{l} \in \mathcal{F}_{\text {nor }}\left(\alpha_{k(l)}\right)$ and of operators $X_{l} \in \mathcal{F}$ such that $\alpha_{k(l)} \cap \operatorname{Sp}\left(A_{j}\right)=0$, for $j<l$, and

$$
\left\|\left[A_{l}^{*}, X_{l}\right]\right\|_{\mathfrak{S}^{1}}=\left\|\left[h\left(A_{l}\right), X_{l}\right]\right\|_{\mathfrak{S}^{1}} \geqslant l^{3}\left\|\left[A_{l}, X_{l}\right]\right\|_{\mathfrak{S}^{1}} .
$$

Now, repeating the proof of Theorem 5.6, we construct operators

$$
A=\overbrace{A_{1} \oplus \cdots \oplus A_{1}}^{m_{1}} \oplus \cdots \oplus \overbrace{A_{n} \oplus \cdots \oplus A_{n}}^{m_{n}} \oplus \cdots
$$

and

$$
Z=\overbrace{Z_{1} \oplus \cdots \oplus Z_{1}}^{m_{1}} \oplus \cdots \oplus \overbrace{Z_{n} \oplus \cdots \oplus Z_{n}}^{m_{n}} \oplus \cdots,
$$

such that $Z \in C(H),[A, Z] \in \mathfrak{S}^{1}$ and $[h(A), Z]=\left[A^{*}, Z\right] \notin \mathfrak{S}^{1}$. We also have that $A$ is a normal operator, $\operatorname{Sp}(A) \subseteq \alpha_{1}$ and that all eigenspaces of $A$, corresponding to non-zero eigenvectors, are finite dimensional. Hence $A$ is compact.

For regular ideals the $J$-stability of a function is equivalent to it being $J$-Lipschitzian.

Corollary 5.10. Let a regular s.n. ideal $J$ be $J^{\phi}$ or $J_{0}^{\phi}$. Let $g$ be a function on $\alpha \subset \mathbb{C}$. If $\alpha$ is weakly $J$-Fuglede, then the following are equivalent:

(i) $g$ is a $J$-Lipschitz function on $\alpha$;

(ii) $g$ is $J$-stable on $\alpha$.

Proof. (i) $\Longrightarrow$ (ii). This follows from Corollaries 4.6 and 5.7. Conversely, by Proposition 5.5, if $g$ is $J$-stable on $\alpha$, it is commutator $J$-stable on $\alpha$. By Theorem 5.6, $g$ is commutator $J$-bounded on $\alpha$. Hence it is $J$-Lipschitzian on $\alpha$.

We summarize below our results for the important class of ideals $\mathfrak{S}^{p}$.

\section{Corollary 5.11.}

(i) The ideals $\mathfrak{S}^{p}$, for $p \in(1, \infty)$, are regular, Fuglede and weakly Fuglede. The ideal $\mathfrak{S}^{1}$ is regular, but neither Fuglede, nor weakly Fuglede. The ideals $\mathfrak{S}^{\infty}$ and $\mathfrak{S}^{b}$ are weakly Fuglede, but neither regular, nor Fuglede ideals. 
(ii) If a set is $\mathfrak{S}^{p}$-Fuglede for some $p \in\{1, \infty, b\}$, it is Fuglede for all $p \in\{1, \infty, b\}$.

(iii) If $p \in(1, \infty)$, then the following are equivalent:

(1) $g$ is $\mathfrak{S}^{p}$-Lipschitzian on $\alpha$;

(2) $g$ is commutator $\mathfrak{S}^{p}$-bounded on $\alpha$;

(3) $g$ is $\mathfrak{S}^{p}$-stable on $\alpha$;

(4) $g$ is commutator $\mathfrak{S}^{p}$-stable on $\alpha$;

(5) $g$ is $\mathfrak{S}^{q}$-Lipschitzian on $\alpha$ for $p_{-} \leqslant q \leqslant p_{+}$(see (3.7));

(6) there is $D=D(\alpha, g, p)>0$ such that, for $X \in B(H)$ and normal $A, B$ with spectra in $\alpha$, the condition $A X-X B \in \mathfrak{S}^{p}$ implies

$$
g(A) X-X g(B) \in \mathfrak{S}^{p} \quad \text { and } \quad\left\|g(A)-g(B){\| \mathfrak{S}^{p}} \leqslant D\right\| A X-X B \|_{\mathfrak{S}^{p}} .
$$

(iv) If a function $g$ is commutator $\mathfrak{S}^{p}$-bounded on $\alpha$ for some $p$ in $\{1, \infty, b\}$, it is commutator $\mathfrak{S}^{q}$-bounded on $\alpha$ for all $q$ in $[1, \infty] \cup b$.

(v) Let $\alpha$ be $\mathfrak{S}^{p}$-Fuglede, for $p \in\{1, \infty, b\}$. The following are equivalent:

(1) $g$ is $\mathfrak{S}^{p}$-Lipschitzian on $\alpha$;

(2) $g$ is commutator $\mathfrak{S}^{p}$-bounded on $\alpha$;

(3) $g$ is commutator $\mathfrak{S}^{1}$-stable on $\alpha$;

(4) there is $D>0$ such that, for $X \in B(H)$ and normal $A, B$ with spectra in $\alpha$, the condition $A X-X B \in \mathfrak{S}^{1}$ implies

$$
g(A) X-X g(B) \in \mathfrak{S}^{1} \quad \text { and } \quad\|g(A) X-X g(B)\|_{\mathfrak{S}^{1}} \leqslant D\|A X-X B\|_{\mathfrak{S}^{1}} .
$$

If $\alpha \subset \mathbb{R}$, then the above conditions are equivalent to the following condition:

(5) $g$ is $\mathfrak{S}^{1}$-stable on $\alpha$.

(vi) Let $\mathfrak{S}^{p}$ - $\operatorname{Lip}(\alpha)$ be the space of all $\mathfrak{S}^{p}$-Lipschitz functions and let $\operatorname{Lip}(\alpha)$ be the space of all Lipschitz (in the usual sense) functions on $\alpha$. Then

$$
\begin{aligned}
& \mathfrak{S}^{b}-\operatorname{Lip}(\alpha) \subseteq \mathfrak{S}^{p}-\operatorname{Lip}(\alpha) \subseteq \mathfrak{S}^{2}-\operatorname{Lip}(\alpha)=\operatorname{Lip}(\alpha) \quad \text { for } p \in[1, \infty] \cup b ; \\
& \mathfrak{S}^{p}-\operatorname{Lip}(\alpha)=\mathfrak{S}^{p^{\prime}}-\operatorname{Lip}(\alpha) \subseteq \mathfrak{S}^{q}-\operatorname{Lip}(\alpha) \quad \text { for } p_{-} \leqslant q \leqslant p_{+} \text {. }
\end{aligned}
$$

\section{Problem 5.12.}

(i) Do the spaces $\mathfrak{S}^{p}$ - $\operatorname{Lip}(\alpha)$ differ for different $p \in(1,2)$ ? A related question: are all functions in $\operatorname{Lip}(\alpha) \mathfrak{S}^{p}$-Lipschitzian, for all $p \in(1, \infty)$ ?

(ii) The space $C^{(1)}(a, b)$ of all continuously differentiable functions on $[a, b]$ is not contained in $\mathfrak{S}^{b}$ - $\operatorname{Lip}(a, b)$ (see $\left.[\mathbf{1 0}]\right)$. On the other hand, $C^{(1)}(a, b) \subset \mathfrak{S}^{2}-\operatorname{Lip}(a, b)$. Do all spaces $\mathfrak{S}^{p}-\operatorname{Lip}(a, b)$, for $p \in(1, \infty)$, contain $C^{(1)}(a, b)$ ? 
Problem 5.13. Let $g$ be a continuous function on $[-1,1]$ and $p \in[1, \infty]$. Let there exist $D>0$ such that $\|g(A)-g(B)\|_{\mathfrak{S}^{p}} \leqslant D\|A-B\|_{\mathfrak{S}^{p}}$, for all self-adjoint $A, B$ in the unit ball of $\mathfrak{S}^{p}$. Is $g \mathfrak{S}^{p}$-Lipschitzian on $[-1,1]$ ?

It is easy to verify that the answer is positive for $p=2$ and $p=\infty$.

Acknowledgements. Edward Kissin is grateful to the Leverhulme Trust for the award of a research fellowship. The authors are grateful to V. I. Burenkov, E. B. Davies, Yu. B. Farforovskaya, R. Kovac, V. I. Ovchinnikov, V. V. Peller and G. Weiss for helpful discussions.

\section{References}

1. A. Abdessemed and E. B. Davies, Some commutator estimates in the Schatten classes, J. Lond. Math. Soc. 39 (1989), 299-308.

2. J. ARAZY, Some remarks on interpolation theorems and the boundness of the triangular projection in unitary matrix spaces, Integ. Eqns Operat. Theory 1/4 (1978), 453-495.

3. H. Bercovici and D. Voiculescu, The analogue of Kuroda's theorem for $n$-tuples, Operat. Theory Adv. Applic. 41 (1989), 57-60.

4. H. Bercovici, L. Kerchy and R. Kovac, Obstructions to diagonalization modulo Lorentz ideals, Pac. J. Math. 191 (1999), 201-206.

5. M. S. Birman And M. Z. Solomyak, Stieltjes double-integral operators, II, Prob. Mat. Fiz. 2 (1967), 26-60 (in Russian).

6. M. S. Birman and M. Z. Solomyak, Stieltjes double-integral operators, III, Prob. Mat. Fiz. 6 (1973), 28-54 (in Russian).

7. K. N. Boyadzhiev, Norm estimates for commutators of operators, J. Lond. Math. Soc. 58 (1998), 739-745.

8. J. L. DALetskit and S. G. Krein, Integration and differentiation of functions of hermitian operators and applications to the theory of perturbations, Am. Math. Soc. Transl. 2 47 (1965), 1-30.

9. E. B. DAvies, Lipschitz continuity of functions of operators in the Schatten classes, $J$. Lond. Math. Soc. 37 (1988), 148-157.

10. Yu. B. FARForovskAYA, Example of a Lipschitz function of selfadjoint operators that gives a non-nuclear increment under a nuclear perturbation, J. Sov. Math. 4 (1975), 426433.

11. C. K. Fong, On normal operators on Banach spaces, Glasgow Math. J. 20 (1979), 163168.

12. I. Ts. Gohberg AND M. G. KReIn, Introduction to the theory of linear non-selfadjoint operators in Hilbert spaces (Nauka, Moscow, 1965).

13. P. Habala, P. HajeK And V. Zizler, Introduction to Banach spaces (Matfyz Press, Karlovy, 1997).

14. D. R. Jocic, Integral representation formula for generalized normal derivations, Proc. Am. Math. Soc. 127 (1999), 2303-2314.

15. B. E. Johnson and J. P. Williams, The range of a normal derivation, Pac. J. Math. $\mathbf{5 8}$ (1975), 105-122.

16. E. Kissin AND V. S. Shulman, On the range inclusion of normal derivations: variations on a theme by Johnson, Williams and Fong, Proc. Lond. Math. Soc. 83 (2001), 176-198.

17. E. Kissin and V. S. Shulman, Classes of operator-smooth functions, I, OperatorLipschitz functions, Proc. Edinb. Math. Soc. 48 (2005), 151-173.

18. F. Kittaneh, On Lipschitz functions of normal operators, Proc. Am. Math. Soc. 94 (1985), 416-418. 
19. S. G. Krein, Yu. I. Brudnyi And E. M. Semenov, Interpolation of linear operators (Nauka, Moscow, 1978).

20. J. Lindenstrauss And L. Tzafriri, Classical Banach spaces, vol. II, Function spaces (Springer, 1979).

21. B. S. Mityagin, An interpolation theorem for modular spaces, Mat. Sb. 66 (1965), 473482 .

22. V. V. PELLER, Hankel operators in the perturbation theory of unitary and selfadjoint operators, Funkzion. Analysis Ego Priloz. 19 (1985), 37-51.

23. V. S. Shulman, On multiplication operators and traces of commutators, Zap. Nauchn. Semin. LOMI 135 (1985), 182-194.

24. V. S. Shulman, Some remarks on the Fuglede-Weiss theorem, Bull. Lond. Math. Soc. 28 (1996), 385-392.

25. G. Weiss, The Fuglede commutativity theorem modulo the Hilbert-Schmidt class and generating functions for matrix operators, I, Trans. Am. Math. Soc. 246 (1978), 193-209. 